\title{
Numerical investigation of the earplug contribution to the low-frequency objective occlusion effect induced by bone-conducted stimulation
}

Kévin Carillo, ${ }^{1, a)}$ Olivier Doutres, ${ }^{1}$ and Franck Sgard ${ }^{2}$

${ }^{1}$ École de technologie supérieure, Montréal, Quebec H3C 1K3, Canada

${ }^{2}$ Institut de recherche Robert-Sauvé en santé et en sécurité du travail, Montréal, Quebec H3A 3C2, Canada

\begin{abstract}
:
The use of earplugs is commonly associated with an increased perception of the bone-conducted part of one's own physiological noise. This phenomenon is referred to as occlusion effect and is most prominent at low frequencies. Several factors influence the occlusion effect, such as the ear anatomy; the bone-conducted stimulation; and the type of occlusion device and its fit, insertion depth, and material properties. The latter factor is of great interest to potentially reduce the occlusion effect of passive earplugs. This paper investigates the mechanism(s) of contribution of earplugs to the objective occlusion effect. A two-dimensional axi-symmetric finite element model of the outer ear is used and investigated in an electro-acoustic framework. Simulation results are shown to compare reasonably well with measurement data, which qualifies the model to study the influence of earplugs on the occlusion effect. Two mechanisms are highlighted: (i) a Poisson effect induced by the normal component of the earcanal wall vibration and (ii) a longitudinal motion caused by the tangential component of the earcanal wall vibration. By varying the geometry of the surrounding tissues, the spatial distribution of the earcanal wall vibration is shown to influence the contribution of the earplug to the occlusion effect. @ 2021 Acoustical Society of America.
\end{abstract}

https://doi.org/10.1121/10.0006209

(Received 18 December 2020; revised 21 July 2021; accepted 22 August 2021; published online 17 September 2021)

[Editor: Hideko Heidi Nakajima]

Pages: 2006-2023

\section{INTRODUCTION}

The use of earplugs (EPs) is commonly accompanied by an increased perception of the bone-conducted part of one's own physiological noise (e.g., one's own voice, chewing, heartbeat, etc.), a phenomenon called occlusion effect (OE). ${ }^{1}$ This phenomenon is most significant at low frequencies $(<1 \mathrm{kHz})$ for shallow occlusion and is deemed to be a notable source of discomfort, especially to workers wearing EPs. ${ }^{2}$ As a consequence, these hearing protection devices may not be worn consistently and/or correctly. ${ }^{2}$ The name "OE" encompasses several descriptions of the phenomenon: (i) objective OE defined as the sound pressure level (SPL) difference measured by placing a microphone at or near the eardrum between occluded and open configuration, ${ }^{3-7}$ (ii) subjective $\mathrm{OE}$ defined as the difference between the open and occluded hearing thresholds, ${ }^{4-6,8}$ and (iii) perceptive $\mathrm{OE}$ related to the discomfort induced by the phenomenon and commonly quantified using questionnaires. ${ }^{9-11}$ The perceptive OE is certainly the most important phenomenon to reduce since it is directly related to the acoustical comfort dimension of EP wearers. Both subjective and perceptive OE involve all bone-conduction pathways, whereas the objective OE only involves the outer ear pathway. Since (i) this study focuses on the alteration of the outer ear pathway

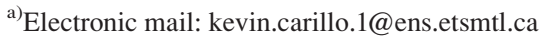

due to the use of EPs and (ii) the outer ear pathway dominates the hearing by bone-conduction when the earcanal $(\mathrm{EC})$ is occluded, ${ }^{12}$ this paper only deals with the objective OE, hereafter simply referred to as the $\mathrm{OE}$ for the sake of conciseness.

At low frequencies, the fundamental mechanism of the $\mathrm{OE}$ induced by an acoustically rigid occlusion of the EC entrance is explained by the acoustic impedance increase from the mass-controlled open EC to the compliancecontrolled occluded EC. This impedance increase is responsible for the augmentation of the sound pressure radiated by the vibrating EC wall in the occluded case compared to the open one. ${ }^{5,8,13-15}$ Several factors are known or are a priori expected to influence the $\mathrm{OE}$ and to explain its large variability documented in the literature: (i) the ear anatomy (e.g., the EC geometry, ${ }^{15}$ the distribution and properties of the tissues surrounding the $\mathrm{EC}^{12,16}$ ); (ii) the position ${ }^{6}$ and nature ${ }^{14}$ of the bone-conducted stimulation; and (iii) the type of occlusion device ${ }^{5,17}$ and its fit, $^{14,18}$ insertion depth, ${ }^{5,19}$ and material properties. ${ }^{7,14,20}$ While the first two groups of factors are intrinsic to the wearer of EPs and cannot be changed in practice, the third set of factors related to the contribution of the $\mathrm{EP}$ on the $\mathrm{OE}$ is of great interest to reduce this phenomenon and increase the acoustic comfort of EPs.

To the authors' knowledge, Bárány ${ }^{3}$ was the first to suggest, in 1938, that the sound pressure generated in the EC occluded by an EP could also be caused by its vibration in 
the EC cavity rather than generated only by the normal vibration of the "free" EC wall (i.e., the portion not covered by the EP). ${ }^{21,22}$ Since then, few studies have been dedicated to understanding the EP influence on the $\mathrm{OE}^{7,14,18,20,23}$ These studies are now discussed.

Hansen $^{14}$ carried out OE experimental measurements on human subjects using a continuous speech, nasal sound, and chewing stimulation for both foam and custom acrylic EPs at medium insertion. The $\mathrm{OE}$ was found to be $5-15 \mathrm{~dB}$ lower for the foam compared to the acrylic EP at low frequencies. To explain the phenomenon, Hansen developed a lumped element model of the occluded EC. As a first step, the acoustical effect of the EP on the EC SPL was modeled as an acoustic impedance, assuming that the sound pressure was solely generated by the free EC wall surrounding the occluded EC cavity. By measuring the acoustic impedance of a foam EP, Hansen showed that its medial surface was seen as an acoustically rigid surface from the occluded EC cavity. Hansen concluded that if a foam EP has an infinite impedance, then so does a silicone EP. Hence, the OE difference between both EPs cannot be explained by the acoustic impedance of their medial surface. Hansen then hypothesized two other mechanisms of contribution of the EP to the occluded EC SPL. In the first mechanism, Hansen $^{14}$ assumed that the EP vibrates as a rigid body (at least at low frequencies) in the longitudinal direction (i.e., EC central axis) depending on its mass (similar to what was suggested by Bárány ${ }^{3}$ ) and on the elasticity of the EC wall surrounding tissues covered by the EP. However, Hansen's model predicted a decrease in the occluded EC SPL when the EP mass increased, whereas the opposite was observed experimentally (the acrylic EP was heavier than the foam EP but generated the greatest $\mathrm{OE}$ ). Hansen concluded that the vibration of the medial EP surface did not contribute to the occluded EC SPL. However, the influence of other EP mechanical properties was not investigated since the EP was considered to be rigid. In the second mechanism, while considering that the whole (open or occluded) EC wall vibration acted as an ideal source of volume velocity, Hansen proposed that the mass of the EP decreased the vibration of the portion of the EC wall coupled to the EP, thus increasing the vibration of the free EC wall (not coupled to the EP). By this mechanism, Hansen's model predicted that a heavy EP caused a higher OE compared to a light EP, such as a silicone EP compared to a foam EP. The role of the EP mass attributed by Hansen to the OE is, however, in contradiction with an experimental work provided by Watson and Gales, ${ }^{24}$ who showed that there is no direct relation between the EP mass and the resulting OE. Also, the assumption proposed by Hansen ${ }^{14}$ considering that the whole EC wall vibration acts as an ideal volume velocity source seems debatable when the EC wall is coupled to the EP. The presence of the EP could rather be expected to decrease the vibration of the whole EC wall.

In an attempt to understand the influence of the EP on the OE, Brummund et al. $^{7}$ investigated the difference in $\mathrm{OE}$ between a foam and a silicone EP at medium insertion, both experimentally and numerically, using a two-dimensional (2D) axi-symmetric FE model of the outer ear that accounts for anatomical tissues surrounding the EC cavity. Simulations showed that most of the acoustic power dissipated at the eardrum was injected by the medial EP surface rather than the free EC wall surface, especially for the silicone EP compared to the foam EP. Brummund et al. concluded that the contribution of the free EC wall was of secondary importance compared to the contribution of the medial EP surface, at least for medium insertion, contrary to what Hansen proposed. However, Brummund et al. did not explain how the EP contributed to the OE.

Lee and Casali $^{20,23}$ carried out an experimental study to examine the influence of the EP type on the OE for shallow and medium insertions. Experimental results showed that the influence of the EP type significantly increased with insertion depth. These results demonstrated that the relative contribution between the free EC wall and the medial EP surface depends on the insertion depth of the EP. Sgard et $a l .{ }^{18}$ observed the increase in the EP influence on the OE with insertion depth using the FE model developed by Brummund et al. ${ }^{7}$ In the limits of the model (discussed in this paper), FE simulations showed that the EP did not significantly influence the vibration of the free EC wall. This emphasized that the influence of the EP on the OE is directly driven by radiation of the medial EP surface in the occluded EC cavity. However, the vibro-acoustic behavior was investigated and interpreted in terms of acoustic power flow in the occluded EC cavity. This approach revealed intricate acoustic power exchanges, in particular between the medial EP surface and the free EC wall, but did not explain the mechanism(s) of contribution of the EP.

This paper is a continuation of previous studies ${ }^{7,18}$ and focuses on the understanding of the influence of the EP on the OE to ultimately be able to modify EP designs to reduce the magnitude of the phenomenon. In particular, this study aims at explaining the difference in OE highlighted in experimental studies ${ }^{7,14,20}$ between a foam and a silicone EP and its dependence on the insertion depth. ${ }^{23}$ For this purpose, an improved version of the 2D axi-symmetric FE model initially developed by Brummund et al. ${ }^{7}$ is proposed. The use of a 2D axi-symmetric truncated model of the outer ear considerably decreases the computation time compared to a realistic $3 \mathrm{D}$ truncated model ${ }^{19}$ and even more in comparison with a whole head model. ${ }^{25,26}$ In addition, the simplified geometry of a 2D axi-symmetric model greatly facilitates the examination of the role of the EP in the objective OE.

To analyze and interpret the FE results, an electroacoustic (EA) framework of the open and occluded EC is developed. The conjunction of both FE model and EA framework allows for a straightforward interpretation of the complex phenomena involved in the vibro-acoustic behavior of such an occluded outer ear excited by bone-conducted stimulation. ${ }^{13}$ Compared to past EA approaches, ${ }^{5,13-15,17,27}$ the medial EP surface is here accounted for as an ideal source of volume velocity, which is distinct from the source 
representing the free EC wall surrounding the occluded EC cavity. Hence, FE simulations are investigated in terms of volume velocity imposed by the free EC wall and the medial EP surface on the occluded EC cavity. The influence of the EP mechanical properties is studied using a design of experiment for shallow, medium, and deep insertion depths. In addition, the influence of the EC wall vibration distribution on the OE is investigated in conjunction with the EP insertion depth by varying the geometry of the EC surrounding tissues. Simulations of the OE are compared to experimental data available in the literature. By examining in detail the vibro-acoustic behavior of the EP using the FE model, this research provides insight about its mechanism(s) of contribution to the objective OE.

\section{FINITE ELEMENT MODEL OF THE OUTER EAR}

\section{A. Geometry}

The three-dimensional (3D) geometry of the open ear, initially developed by Brummund et al. ${ }^{7}$ from literature data, is illustrated in Fig. 1(a). Since the problem is considered axi-symmetric, only a cross-sectional cut through the $(r, z)$ plane is depicted in Fig. 1(b). The outer ear consists of a cylindrical EC of length $l_{E C}$ and radius $r_{E C}$ surrounded by soft (cartilage and skin) and bony tissues. The anatomical landmarks of the pinna are not accounted for in the current model, mainly to preserve the axi-symmetry of the problem. The geometry of the EC entrance region is thus simplified to a $2 \mathrm{~mm}$ protrusion from $z=0$ made of cartilaginous and skin tissues. The eardrum is assumed to mark the end of the cylindrical EC perpendicular to its central axis.

Compared to the original model developed by Brummund et al., ${ }^{7}$ the thickness of the skin surrounding the EC cavity varies linearly from the eardrum to the EC entrance instead of changing abruptly at the bony part/ cartilaginous part transition. In addition, the edge of the skin and cartilaginous tissues at the EC entrance has been radiused in order to not couple the EP with the protruding extension representing the pinna in the occluded case. In a real ear, the transition between soft and bony tissues along the EC is not very clear and varies gradually around the 3D EC cavity from the EC entrance to the eardrum. This transition is estimated to occur approximately at the second bend of the EC, close to its half-length. ${ }^{28,29}$ In the current model, a "straight" transition of the underlying tissues is assumed at the EC half-length, as used by Brummund et al. ${ }^{7}$ In addition, to study the influence of a variation of the $\mathrm{EC}$ wall vibration distribution on the contribution of the EP to the OE, two other simplified anatomical geometries ["slanted" and "curved"; see Figs. 1(c) and 1(d), respectively] of the EC surrounding tissues including gradual transitions along both radial and longitudinal axis are also used (see Sec. III C). These additional transitions have been determined from a 3D anatomical model constructed by Brummund et al. ${ }^{19}$ based on cryosection images of a human female cadaver from the Visible Human Project ${ }^{\circledR}$. Figure 2 displays the 3D anatomical model developed by Brummund et al. ${ }^{19}$ in subplot (a), the slanted transition in subplot (b), and both straight and curved transition in subplot (c). An example of (a)
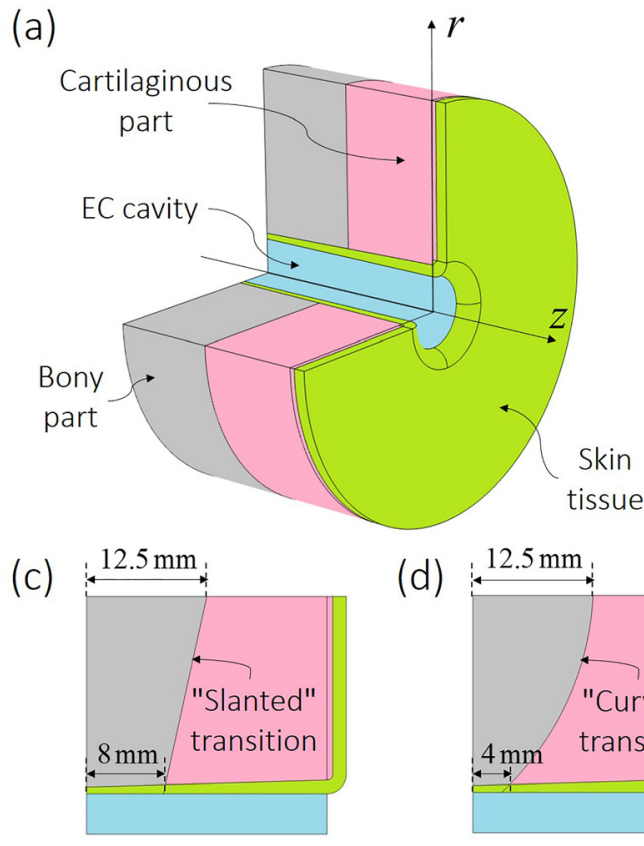

(d)
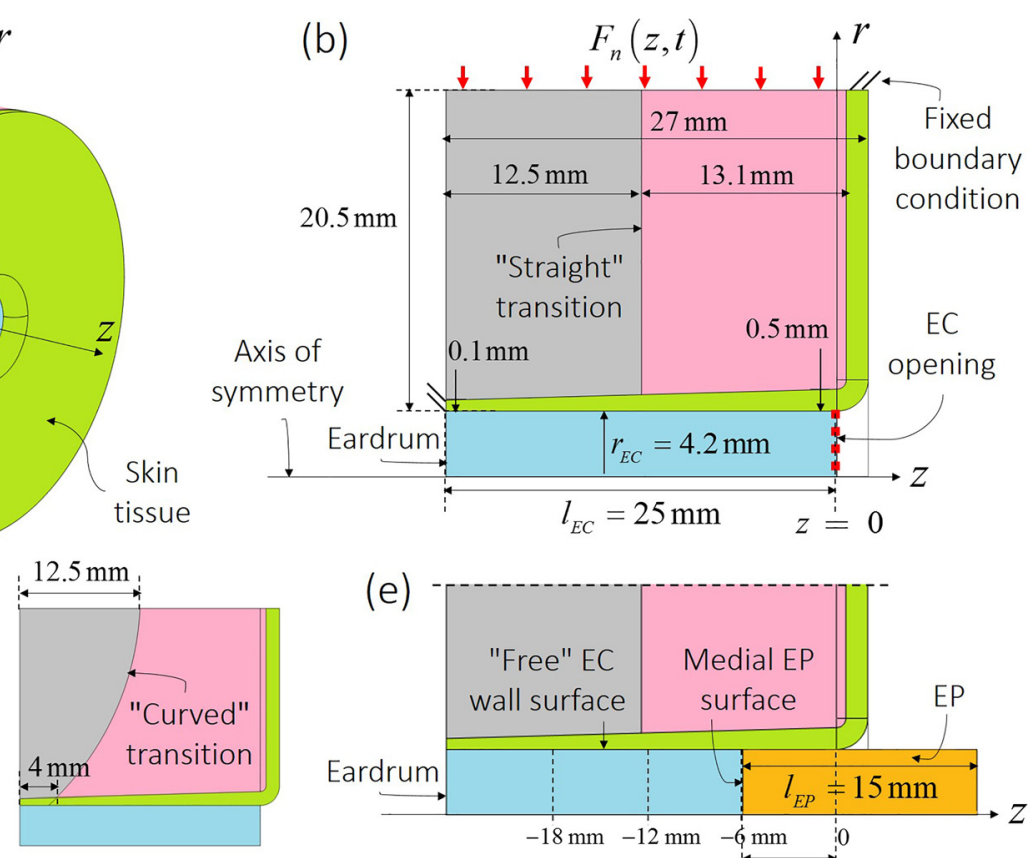

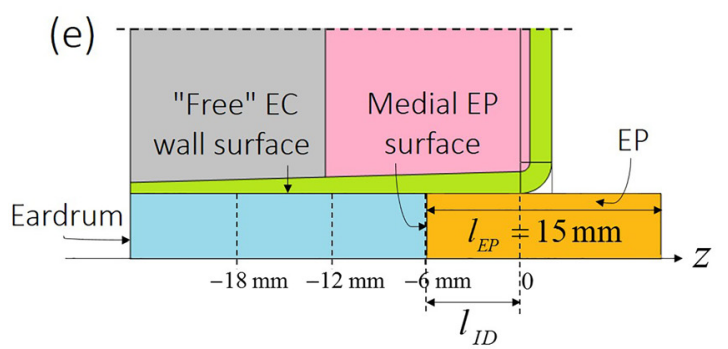

FIG. 1. (Color online) 3D view of the axi-symmetric FE model of an outer ear (a) and 2D view in the $(r, z)$ plane of the open EC (b) with different transitions between cartilaginous and bony tissues [(c) and (d)] and 2D view of the occluded EC (e). In (b), the red dashed line at $z=0$ indicates the EC opening, the red arrows at the top of the model indicate where the normal force $F_{n}(z, t)$ is exerted, and the double slash indicates fixed constraint. In (e), the drawing of the model has been cut along the $z$ axis (see the dashed black line) to limit the size of the figure. 

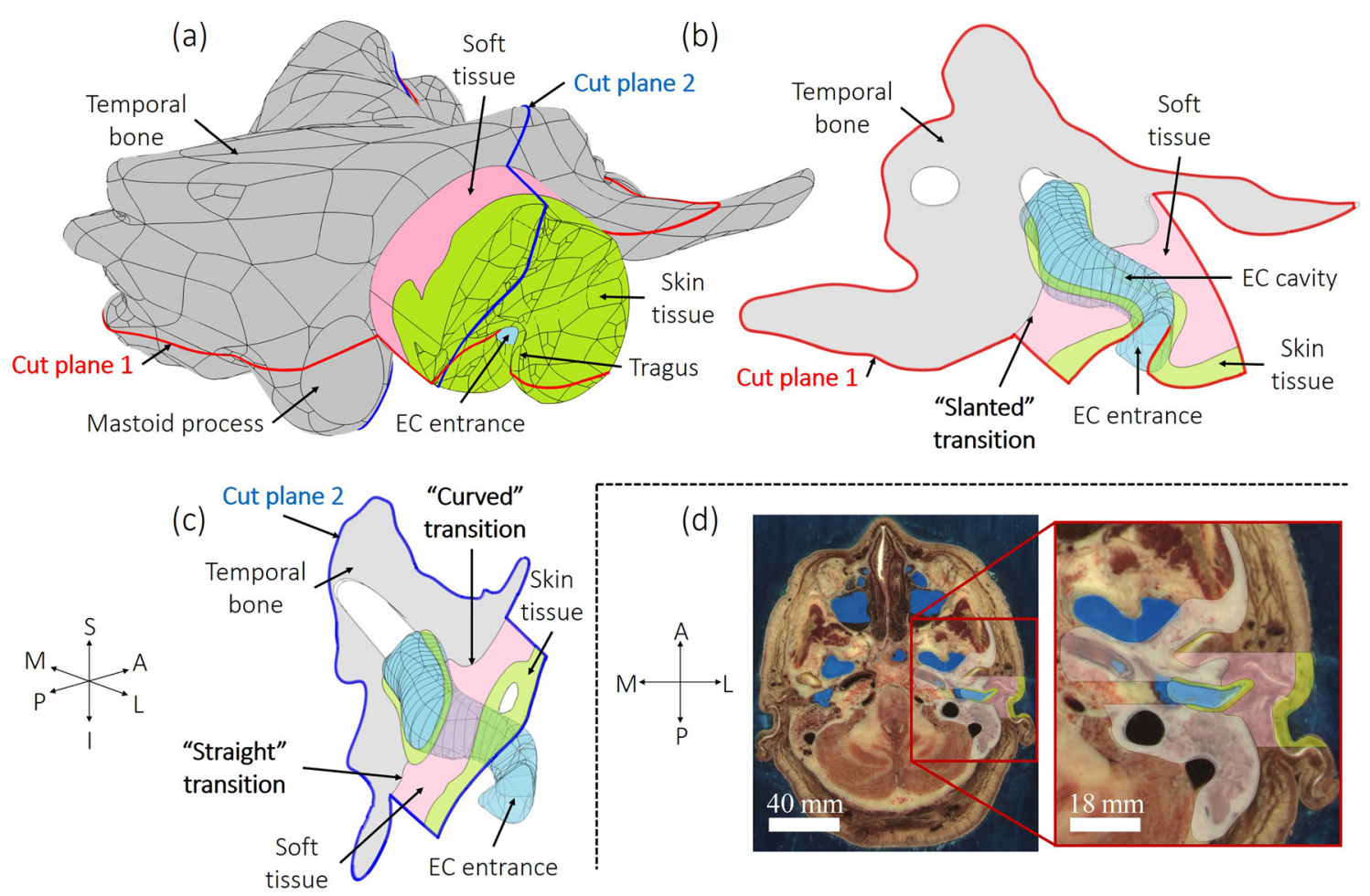

FIG. 2. (Color online) 3D anatomical model of a human outer ear developed by Brummund et al. (Ref. 19) (a); sectional view of the model in cut plane 1 (b) and cut plane 2 (c); and example of a cryosection image from the Visible Human Project ${ }^{\circledR}$ used to reconstruct the 3D anatomical model (d). Cut planes 1 and 2 are perpendicular to a same EC cross-sectional plane located close to the second bend of the EC. (b) shows a slanted transition between bony and cartilaginous tissues, whereas (c) shows straight and curved transitions. The coordinate system refers to superior (S), inferior (I), posterior (P), anterior (A), medial (M), and lateral (L).

a cryosection image superimposed on a sectional view of the 3D model is shown in Fig. 2(d). Figure 2 illustrates the geometrical complexity of the anatomical tissues surrounding the EC. In the current 2D FE model, this complexity is simplified due to the axi-symmetrical assumption.

The axi-symmetric geometrical representation of the EC occluded by an EP is depicted in Fig. 1(e). Two EPs are considered: foam and silicone. Their length is equal to $15 \mathrm{~mm}$, which is in between a push-to-fit foam EP (about $10 \mathrm{~mm}$ length) and a roll-down foam EP (19 mm length). The silicone EP represents a custom molded EP. This selection of two EPs identical except for their composition allowed for studying the influence of the EP material properties on the OE. Furthermore, the diameter of each EP is identical and selected to match exactly the inside diameter of the EC. Therefore, no deformation of the EC surrounding tissues due to the presence of the EP is taken into account. In the case of the roll-down foam EP, its insertion in the EC induces a radial deformation, which is accounted for by using equivalent mechanical properties (see Sec. II B). In the case of the custom molded silicone EP, it is assumed here to not deform. In practice, however, custom molded EPs are slightly larger than the EC imprints from which they are made in order to fit tightly and seal the EC. This is likely to deform the EC surrounding tissues a certain amount, ${ }^{31}$ but this phenomenon is not accounted for here due to the lack of knowledge of the deformation and the prestress material properties of the EC surrounding tissues.
Three insertion depths, defined from the EC entrance to the medial EP surface, are considered here: shallow $(6 \mathrm{~mm})$, medium $(12 \mathrm{~mm})$, and deep $(18 \mathrm{~mm})$. These insertion depths are consistent with the literature. Berger and Kerivan ${ }^{8}$ used partial (shallow) insertion at about $5 \mathrm{~mm}$, standard (medium) insertion at about $10 \mathrm{~mm}$, and deep insertion at about $19 \mathrm{~mm}$. Stenfelt and Reinfeldt ${ }^{5}$ and Brummund et al. ${ }^{19}$ used slightly longer insertion depths (i.e., a $7 \mathrm{~mm}$ shallow insertion and a $22 \mathrm{~mm}$ deep insertion). For the $\operatorname{EAR}^{\circledR}$ Classic $^{\circledR}$ EP of 19 mm length, Berger ${ }^{30}$ defined the partial (shallow) insertion as about $15 \%-20 \%$ of the EP inserted in the EC, the standard (medium) insertion as about $50 \%-60 \%$ of the EP in the EC, and the deep insertion as about $80 \%-100 \%$ of the EP inserted in the EC. Considering the shorter length of the EP considered here, the resulting insertion depths are equivalent in this study.

\section{B. Materials}

Modeling the OE using the FE method requires knowledge of the effective material properties of the EC surrounding tissues, which are difficult to assess experimentally, and that of the EP. Similar to other FE models of the outer ear, ${ }^{13,19,25,26}$ solid domains are assumed to exhibit linear elastic isotropic material behavior with constant structural loss factor. Material properties come from previously published experimental data detailed in Refs. 7 and 19 (except for structural loss factors, which were assumed) and 
summarized in Table I (see Appendix A). In practice, the EC surrounding tissues are more likely to be nonlinear, viscoelastic, and anisotropic materials. However, as mentioned previously, the lack of experimental knowledge about human tissues' mechanical properties limits the accuracy of the EC surrounding tissue modeling. Also, the effective material properties of soft tissues could be influenced by the deformation of the EC wall induced by the coupling with the EP in the occluded case, depending on the EP type, ${ }^{31}$ but soft tissues' deformation is not accounted for in the current model as can be understood from the discussion in Sec. II A.

Foam material properties depend on the EP radial strain. $^{32}$ Considering the small EAR Classic ${ }^{\circledR}$ (diameter around $12.3 \mathrm{~mm}$ ), its insertion in the current EC (diameter of $8.4 \mathrm{~mm}$ ) leads to a radial strain of the EP around $31 \%$. James $^{32}$ determined the equivalent (i.e., effective) Young's modulus, density, and Poisson's ratio of a foam EP for several radial strains: $6 \%, 18 \%$, and $30 \%$. Here, material properties of the foam EP were therefore taken to correspond to the $30 \%$ radial strain to account for its prestress in the EC. In addition, the isotropic structural loss factor of the foam EP (small EAR Classic ${ }^{\circledR}$ ) has been measured using a quasistatic mechanical analyzer ${ }^{33}$ for a longitudinal compression rate equal to $30 \%$. The material properties of the silicone EP were taken from experimental data used by Sgard et al. ${ }^{34} \mathrm{In}$ the frequency range of interest, both Young's modulus and loss factor do not significantly vary with frequency and are thus taken as constant. ${ }^{35}$ Material properties of foam and silicone EPs are summarized in Table I (see Appendix A).

The air within the EC cavity is assumed to be a perfect compressible fluid of density $\rho_{0}=1.2 \mathrm{~kg} \cdot \mathrm{m}^{-3}$ and sound speed $c_{0}=343 \mathrm{~m} \cdot \mathrm{s}^{-1}$. Viscous and thermal losses at the fluid/structure boundary are not accounted for since they do not significantly influence the acoustic pressure or the volume velocity transfer in the EC cavity between $100 \mathrm{~Hz}$ and $1 \mathrm{kHz} .^{13}$

\section{Boundary and loading conditions}

The coupling between the EC cavity of the open ear and the surrounding environment is modeled as a locally reacting acoustic impedance boundary condition defined at the EC entrance, similarly to other models. ${ }^{5,7,13,17,19}$ The acoustic radiation impedance of the EC entrance depends on the geometry of the pinna, on the size of the EC entrance, and on scattering effects induced by the head and the body. This impedance is here assumed equal to the radiation impedance $\hat{Z}_{\text {rad }}$ (the caret symbol over $Z$ represents a complex value) of a baffled circular piston of the same area as the EC entrance. ${ }^{13}$ At low frequencies, this assumption provides a reasonable approximation of the measured acoustic radiation impedance of the EC entrance. ${ }^{36}$ The sound radiation of the external tissue in the open EC is however neglected. This effect can be accounted for by modeling the external air environment surrounded by a perfectly matched layer (simulating the Sommerfeld radiation condition) ${ }^{18}$ but has little influence on the open EC SPL in the current model (simulation not shown here) due to its truncation.

The middle and inner ears are accounted for as a locally reacting acoustic impedance $\hat{Z}_{T M}$ boundary condition specified at the eardrum and defined by Shaw and Stinson's model. ${ }^{37}$ This impedance compares well to measurement data at low frequencies. ${ }^{36}$ This assumption is a simplification in regard to the sound radiation that stems from the inertial movement of the ossicles in the middle ear. However, the SPL in open and occluded ECs is not expected to be significantly influenced by the acoustic radiation of the tympanic membrane induced by the vibration of the middle ear ossicles excited via their ligaments by the boneconducted propagation, at least at low frequencies below the middle ear resonance. ${ }^{12,17,38}$ Another argument supporting this assumption is that the OE would not decrease with insertion depth if the tympanic membrane was a significant source of SPL generated in the EC. ${ }^{14}$ The bone-conducted pathway through the middle ear rather influences the vibration transmitted to the cochlea and therefore influences the subjective OE. ${ }^{4,5,17}$ This paper, however, only focuses on the objective OE (i.e., difference in SPL generated in occluded and open cases) and in particular on the contribution of EPs to the SPL generated in the occluded EC. Finally, fixed boundary conditions applied to solid domains of the FE model are indicated by double slashes in Fig. 1(b), whereas remaining solid boundaries are free.

The structure-borne mechanical boundary load is introduced as a uniform normal force field $F_{n}(z, t)=\Re\left[A(z) e^{j \omega t}\right]$ applied to the cartilaginous and bony tissue circumferential boundary, where $A(z)=1 \mathrm{~N}\left(-l_{E C} \leq z \leq 0\right), j$ is the imaginary number, $\omega$ is the circular frequency such that $\omega=2 \pi f$ with $f$ the frequency, and $t$ represents the time. It should be noted that this loading is an idealization of the real stress vectors that act on the human tissues and likely vary in terms of amplitude, phase, and direction along the tissue boundaries. The resulting vibration of the EC wall is thus analyzed in Sec. III B.

\section{Finite element modeling}

A temporal dependency $e^{j \omega t}$ for all fields is assumed henceforth. The sound propagation in solid domains is governed by the classic linear elasto-dynamic equation. ${ }^{39}$ Continuity of stress vectors and displacements is assumed between solid domains. The sound propagation within the air-filled EC cavity is governed by the homogeneous Helmholtz equation $\nabla^{2} \hat{p}+k_{0}^{2} \hat{p}=0$, where $k_{0}=\omega / c_{0}$ is the lossless wavenumber. The coupling at the EC wall/cavity boundary is achieved by the continuity of normal component velocity vectors and normal component stress vectors. Finally, the coupled FE model is meshed according to a criterion of at least six 6-noded quadratic triangular elements per wavelength $\lambda=c / f$ at $1 \mathrm{kHz}$. In a solid, both compression waves and shear waves can propagate. The shortest wavelength has to be taken at the highest frequency of interest (i.e., $1 \mathrm{kHz}$ ) to determine the size of elements allowing 
for achieving convergence. Sound speeds associated with compression and shear waves are, respectively, defined in $2 \mathrm{D}$ by $c_{L}=\sqrt{E /[2 \rho(1-\nu)]}$ and $c_{T}=\sqrt{E /[2 \rho(1+\nu)]}$, with the Young's modulus $E$, the density $\rho$, and the Poisson's ratio $\nu$ of a given solid domain. The less stiff domains, such as skin tissue, are thus the most finely meshed. The mesh of the whole model consists of approximately 4500 elements depending on the configuration and allows for convergence of the solution. This coupled elastoacoustic FE model is solved using COMSOL Multiphysics 5.5 (COMSOL, Stockholm, Sweden). Displacement fields in the tissues and the acoustic pressure field in the EC cavity are computed at each node of the mesh.

\section{E. Vibro-acoustic indicators and framework of interpretation}

The OE is computed from the acoustic pressure $\left\langle\hat{p}_{T M}^{k}\right\rangle$, where $k \in\{$ open, occl $\}$, calculated at the tympanic membrane (surface averaged) in open and occluded cases such as

$$
O E=20 \log _{10}\left(\left|\frac{\left\langle\hat{p}_{T M}^{o c c l}\right\rangle}{\left\langle\hat{p}_{T M}^{\text {open }}\right\rangle}\right|\right) .
$$

Brummund et al. ${ }^{7,19}$ proposed to investigate the $\mathrm{OE}$ computed using FE models based on an acoustic power balance approach. However, acoustic power exchanges can be intricate to investigate (i) the relative contribution between the free EC wall and the medial EP surface and (ii) the influence of the EP material on the OE. Hence, in the current paper, a framework of investigation based on EA analogy is provided to dissect and interpret the OE computed using the FE model.

According to the EA framework, when the EC is open, the vibration of the EC wall acts as an ideal source of volume velocity $\hat{q}_{\text {wall }}^{\text {open }}$ defined by

$$
\hat{q}_{\text {wall }}^{\text {open }}=\left\langle\hat{v}_{n, \text { wall }}^{\text {open }}\right\rangle S_{\text {wall }}^{\text {open }} \text {, }
$$

with $\left\langle\hat{v}_{n \text {,wall }}^{\text {open }}\right\rangle$ the surface averaged EC wall normal velocity computed using the FE model and $S_{\text {wall }}^{\text {open }}$ the whole EC wall area. The acoustic pressure generated at the tympanic membrane by the $\mathrm{EC}$ wall vibration is then given by

$$
\hat{p}_{T M}^{\text {open }}=\hat{Z}_{E C}^{\text {open }} \times \hat{q}_{\text {wall }}^{\text {open }},
$$

where $\hat{Z}_{E C}^{\text {open }}$ is the acoustic impedance of the open EC cavity seen by the volume velocity source representing the $\mathrm{EC}$ wall normal vibration. The acoustic impedance $\hat{Z}_{E C}^{\text {open }}$ can be approximated in the low frequency range by ${ }^{13}$

$$
\hat{Z}_{E C}^{\text {open }} \approx j \omega\left(L_{E C}^{\text {open }}+L_{\text {rad }}\right),
$$

with $L_{E C}^{\text {open }}=-\rho_{0} l_{c, n} /\left(\pi r_{E C}^{2}\right)$ the acoustic mass of the open EC cavity and $L_{r a d}=8 \rho_{0} /\left(3 \pi^{2} r_{E C}\right)$ the acoustic mass of radiation of the EC opening considered as a baffled circular piston. The open EC acoustic mass $L_{E C}^{\text {open }}$ depends on the centroid position $l_{c, n}$ of the EC wall normal velocity. ${ }^{13}$
In Appendix B, this dependence is mathematically justified. In the FE model, the centroid position $l_{c, n}$ is computed by

$$
l_{c, n}=\frac{\int_{-l_{E C}}^{0} z\left|\hat{v}_{n, \text { wall }}^{\text {open }}(z)\right| \mathrm{d} z}{\int_{-l_{E C}}^{0}\left|\hat{v}_{n, \text { wall }}^{\text {open }}(z)\right| \mathrm{d} z},
$$

where the $z$ axis represents the EC curvilinear axis in the current 2D axi-symmetric FE model. In the current paper, a centroid position $l_{c, t}$ is similarly defined by Eq. (5) but uses the tangential component $\hat{v}_{t, \text { wall }}^{\text {open }}$ of the EC wall velocity instead of the normal component $\hat{v}_{n, \text { wall }}^{\text {open }}$.

When the EC is occluded, the free EC wall (portion of the EC not covered by the EP) and the medial EP surface impose on the EC cavity the volume velocities $\hat{q}_{\text {wall }}^{\text {occl }}$ and $\hat{q}_{E P}$, respectively. The volume velocity imposed by the free EC wall is defined by

$$
\hat{q}_{\text {wall }}^{\text {occl }}=\left\langle\hat{v}_{n, \text { wall }}^{\text {occl }}\right\rangle S_{\text {wall }}^{o c c l}
$$

with $\left\langle\hat{v}_{n, \text { wall }}^{\text {occl }}\right\rangle$ the surface averaged EC wall normal vibration computed using the FE model in the occluded case and $S_{\text {wall }}^{o c c l}$ the free EC wall area. The volume velocity imposed by the medial EP surface is defined by

$$
\hat{q}_{E P}=\left\langle\hat{v}_{n, E P}\right\rangle S_{E P},
$$

with $\left\langle\hat{v}_{n, E P}\right\rangle$ the surface averaged normal vibration of the medial EP surface computed using the FE model and $S_{E P}$ the medial EP surface area. Using the superposition principle, the acoustic pressure at the tympanic membrane in the occluded case is then given by

$$
\hat{p}_{T M}^{o c c l}=\hat{Z}_{E C}^{o c c l} \times\left(\hat{q}_{\text {wall }}^{o c c l}+\hat{q}_{E P}\right),
$$

where $\hat{Z}_{E C}^{o c c l}$ is the acoustic impedance of the occluded EC cavity. The acoustic impedance $\hat{Z}_{E C}^{\text {occl }}$ can be approximated in the low frequency range by ${ }^{13}$

$$
\hat{Z}_{E C}^{o c c l} \approx\left[j \omega\left(C_{E C}^{o c c l}+C_{T M}\right)\right]^{-1}
$$

where $C_{E C}^{o c c l}=\pi r_{E C}^{2}\left(l_{E C}-\left|l_{I D}\right|\right) /\left(\rho_{0} c_{0}^{2}\right)$ represents the acoustic compliance of the occluded EC cavity, which depends on the insertion depth $l_{I D}$, and $C_{T M}$ represents the acoustic compliance of the tympanic cavity volume equal here to $0.78 \mathrm{~cm}^{3}$ (Ref. 13). It is noteworthy that the simplification of the eardrum acoustic impedance to the acoustic compliance $C_{T M}$ is accurate below approximately $500 \mathrm{~Hz} .^{13}$

Finally, by substituting Eqs. (3) and (8) into Eq. (1), the OE computed using the FE model can be approximated in the EA framework by

$$
O E \approx 20 \log _{10}\left[\frac{\hat{Z}_{E C}^{\text {occl }} \times\left(\hat{q}_{\text {wall }}^{\text {occl }}+\hat{q}_{E P}\right)}{\hat{Z}_{E C}^{\text {open }} \times \hat{q}_{\text {wall }}^{\text {open }}}\right] .
$$




\section{RESULTS AND DISCUSSION}

This section is organized as follows. In Sec. III A, the simulations of OE computed using the FE model are compared to corresponding experimental measurements on human subjects available in the literature to assess the realism of the model. In Sec. III B, the contribution of EPs to the $\mathrm{OE}$ is analyzed as a function of its insertion depth and its material properties. In Sec. III C, the influence of the distribution of the EC wall vibration on the $\mathrm{OE}$ and in particular on the contribution of EPs is studied. Finally, Sec. III D discusses the limits of the current study.

\section{A. Evaluation of the finite element simulations with respect to experimental data}

Figure 3 displays FE simulations of the OE paired with experimental data of the objective $\mathrm{OE}$ taken from the literature. Figure 3(a) shows the OEs of the shallowly $(6 \mathrm{~mm})$ and
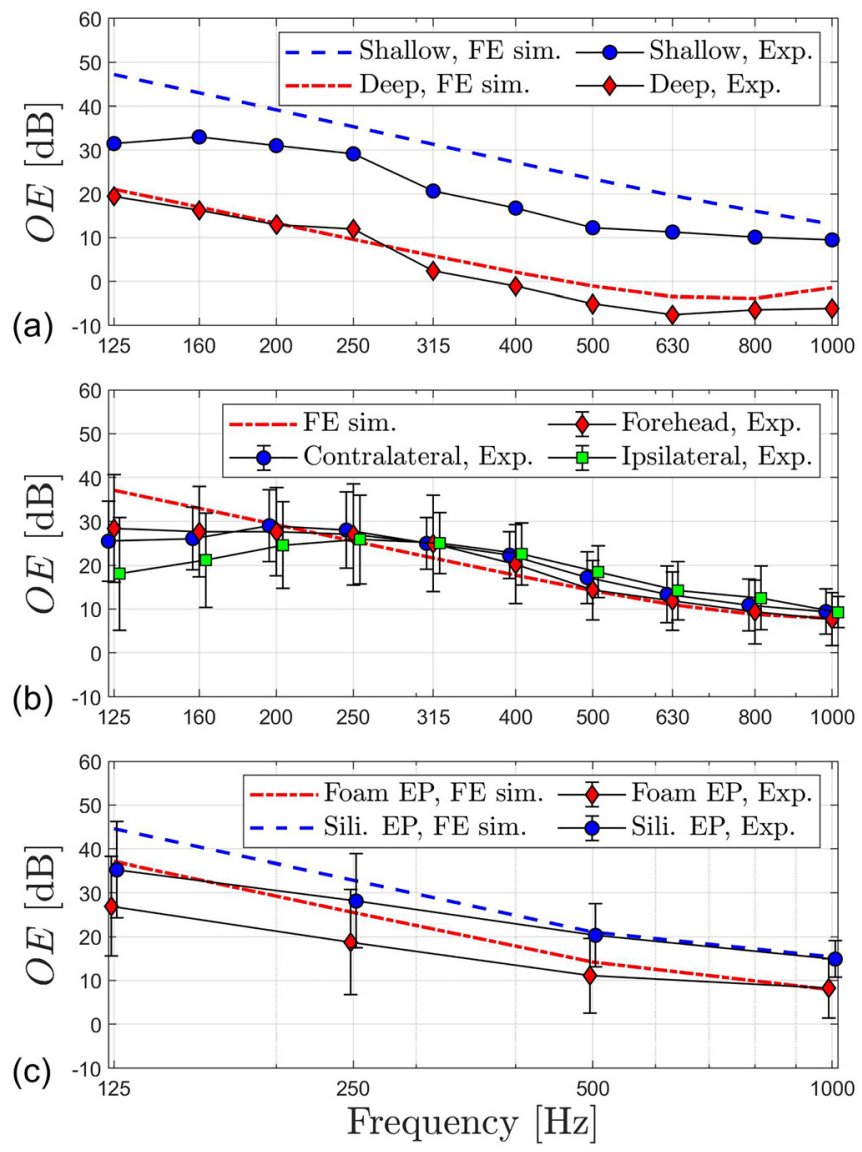

FIG. 3. (Color online) Comparison of predicted and measured objective OE for various conditions. (a) OEs induced by a foam EP at shallow and deep insertion computed using the FE model versus experimental data (median value only) measured on human subjects by Stenfelt and Reinfeldt (Ref. 5) (third octave bands). (b) OEs induced by a foam EP at medium insertion computed using the FE model versus experimental data (mean and associated standard deviation) measured on human subjects by Reinfeldt et al. for contralateral, ipsilateral, and forehead stimulation (Ref. 6) (third octave bands). (c) OEs induced by a foam EP and a silicone EP at medium insertion computed using the FE model versus experimental data (mean and associated standard deviation) measured by Brummund et al. (Ref. 7) (third octave bands). deeply $(18 \mathrm{~mm})$ inserted foam EP computed using the FE model as a function of frequency (third octave bands) in comparison with the median values of experimental data measured by Stenfelt and Reinfeldt ${ }^{5}$ on 20 human subjects. Means and associated standard deviations were not available in Ref. 5. In the measurements, the bone-conducted stimulation was ensured by a bone-transducer applied at the center of the forehead. The OE was computed as the difference in SPLs measured in the occluded and open EC using a probe tube microphone. The shallow and deep insertion of the foam EP corresponded, respectively, to 7 and $22 \mathrm{~mm}$ from the EC entrance. According to Fig. 3(a), the greatest experimental $\mathrm{OE}$ is observed for shallow insertion and begins close to $35 \mathrm{~dB}$ at $125 \mathrm{~Hz}$ and then decreases to $9 \mathrm{~dB}$ at $1 \mathrm{kHz}$. For deep insertion, the mean of experimental OE is $15 \mathrm{~dB}$ lower than for shallow insertion for the whole frequency range. The model overestimates by $4-15 \mathrm{~dB}$ the median experimental $\mathrm{OE}$ for shallow insertion, while both simulated and median experimental OEs are in good agreement for deep insertion.

In complement to Fig. 3(a), Fig. 3(b) shows the OE induced by the foam EP at medium insertion $(12 \mathrm{~mm})$ computed using the FE model as a function of frequency (third octave bands) and compared to experimental data (means and associated standard deviations) obtained by Reinfeldt et $a l .{ }^{6}$ on 20 human subjects. In the measurements, three positions for the bone-transducer were considered: (i) at the center of the forehead (similar to Stenfelt and Reinfeldt ${ }^{5}$ ), (ii) at the contralateral mastoid, and (iii) at the ipsilateral mastoid. The OE was measured using a probe tube microphone inserted close to the eardrum. The occlusion was ensured by a foam EP inserted at $10 \mathrm{~mm}$ relative to the EC opening. The current FE model could account for the influence of the stimulation position by using a set of mechanical boundary conditions and loading other than the set defined in Sec. II C. However, the present work focuses on the influence of EP material on the OE. Hence, the influence of the stimulation position is not of primary importance here. Furthermore, according to Fig. 3(b), the simulated OE lies within one standard deviation associated with the mean experimental data for all stimulation positions above $200 \mathrm{~Hz}$. Below this frequency, the model overestimates the mean experimental $\mathrm{OEs}$ by $10 \mathrm{~dB}$ (forehead stimulation) to $19 \mathrm{~dB}$ (ipsilateral stimulation) at $125 \mathrm{~Hz}$.

Finally, Fig. 3(c) shows the OEs provided by the medium insertion $(12 \mathrm{~mm})$ of the foam and the silicone EPs computed using the FE model as a function of frequency (third octave bands) in comparison with experimental data (means and associated standard deviations) measured by Brummund et al. ${ }^{7}$ in a group of 37 participants. In the measurement, the bone-transducer was placed on the mastoid process for ipsilateral stimulation, and the SPL was measured in open and occluded EC using miniature microphone. Foam and custom molded silicone EP were, respectively, inserted into the EC at $11 \pm 3$ and $12 \pm 1 \mathrm{~mm}$ from the EC entrance of participants. According to Fig. 3(c), the greatest experimental $\mathrm{OE}$ is observed for the silicone EP and begins 
close to $35 \mathrm{~dB}$ at $125 \mathrm{~Hz}$ and then decreases to $15 \mathrm{~dB}$ at $1 \mathrm{kHz}$. The mean experimental OE induced by the foam EP is $8 \mathrm{~dB}$ lower than the silicone EP for the whole frequency range. For a similar insertion depth, Lee and Casali ${ }^{23}$ found the same difference in OE between a foam EP and a medical balloon-based EP (made of silicone and inflated after insertion) measured on six human subjects at $500 \mathrm{~Hz}$ (third octave band). ${ }^{23}$ For both EPs, the variability of experimental data decreases with frequencies above $250 \mathrm{~Hz}$ [see Fig. 3(c)]. The model overestimates the mean experimental OEs induced by foam and silicone EPs by around $9 \mathrm{~dB}$ at $125 \mathrm{~Hz}$ and $5 \mathrm{~dB}$ at $250 \mathrm{~Hz}$. However, the simulated OEs lie within one standard deviation of the mean of the experimental data. The discrepancy between simulated and mean experimental OEs then vanishes at $500 \mathrm{~Hz}$ and $1 \mathrm{kHz}$.

The decrease with frequency of the OE (see Fig. 3) is explained by the change in the character of the acoustic impedance between the open EC, controlled by its acoustic mass [see Eq. (4)], and the occluded EC, controlled by its compliance [see Eq. (9)]. 5,13,14 The FE simulations provide this characteristic slope of the $\mathrm{OE}$ but tend to overestimate the OE below $250 \mathrm{~Hz}$, particularly for shallow [see Fig. 3(a)] and medium insertion [see Figs. 3(b) and 3(c)]. This overestimation could be explained by an incomplete seal between the EP and the EC wall during OE measurements. ${ }^{14,19}$ An incomplete seal is indeed more likely to occur when the EP is not deeply inserted in the EC. The effect of an incomplete seal is not accounted for in this work but has been investigated by the authors in Ref. 18 and corroborates the previous observation that it could be responsible for a reduction of the OE below $250 \mathrm{~Hz}$.

Also, the simplifications of the current model in terms of geometry, boundary conditions, and loading could explain the difference in OE between simulations and experimental data below $250 \mathrm{~Hz}$. First, the current model does not account for the acoustic radiation of external tissues, which could influence the SPL in the open EC. ${ }^{40}$ This phenomenon could be accounted for using a whole head model surrounded by the external air environment and bounded by a perfectly matched layer. ${ }^{18}$ Second, the current model does not differentiate stimulation positions (e.g., forehead, contralateral mastoid, and ipsilateral mastoid) due to its truncation, whereas this factor is shown to influence the experimental OE mainly below $250 \mathrm{~Hz}$ (Ref. 6) [see Fig. 3 (b)]. Whole head models ${ }^{25,26}$ could be a great candidate to account for the influence of both the acoustic pathway through the open EC entrance and the stimulation position, which are not studied here. The most important discrepancy between simulation and measurement occurs for the shallowly inserted foam EP [see Fig. 3(a)]. In this case, the FE model overestimates by $15 \mathrm{~dB}$ the median value of experimental data but fits between the extrema of measured OEs ${ }^{5}$ (not presented here). However, the model does not necessarily represent the median subject of experimental data but rather a particular subject. In other cases, the FE model compares well with measurements (above $250 \mathrm{~Hz}$ ). In particular, the FE model accurately predicts the difference in
OE between a foam and a silicone EP [see Fig. 3(c)], which makes it possible to use this model to investigate the role of the EP in the OE.

\section{B. Contribution of the EP to the OE}

Figure 4 displays the OE induced by a foam EP and a silicone EP at (a) shallow (6 mm), (b) medium (12 mm), and (c) deep $(18 \mathrm{~mm})$ insertions computed using the FE model. It is noteworthy that part of the FE simulations displayed in Fig. 4 in narrowband have already been presented in third octave bands in Fig. 3.

In Fig. 4, the OE induced by the infinite impedance represents a reference case in which the contribution of the "EP" in terms of volume velocity to the OE is zero. This configuration is used to quantify the OE induced only by the vibration of the free EC wall. It is important to note that the differences in OE across the various insertion depths and impedances observed in Fig. 4 are solely due to the vibroacoustic response of the occluded EC because the open EC is the same for all simulations of the OE. These differences in $\mathrm{OE}$ depend on the insertion depth of the occlusion, investigated in Sec. III B 1, and on the type of occlusion (infinite impedance, foam, or silicone EP), studied in Sec. III B 2. In the following, FE simulations of the OE displayed in Fig. 4 are investigated and interpreted in the EA framework (presented in Sec. II E). For this purpose, the accuracy of the EA
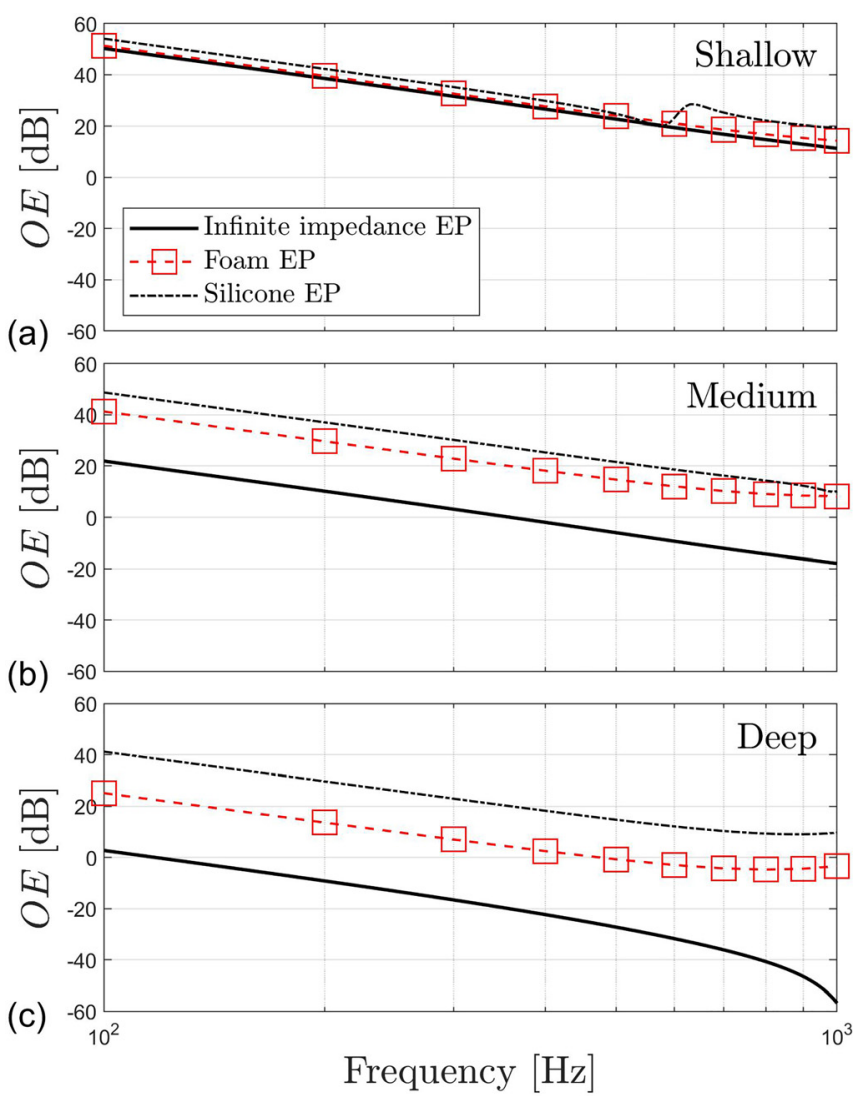

FIG. 4. (Color online) Predicted objective OE induced by an infinite impedance, a foam EP, and a silicone EP at (a) shallow $(6 \mathrm{~mm})$, (b) medium $(12 \mathrm{~mm})$, and (c) deep $(18 \mathrm{~mm})$ insertion computed using the FE model. 
framework to approximate FE simulations of the objective $\mathrm{OE}$ is verified in Fig. 10 (see Appendix C).

\section{Influence of the EP insertion depth}

According to Fig. 4, the OE decreases with the insertion depth for all occlusions (infinite impedance, foam EP, and silicone EP). In the EA framework, Eq. (10) indicates that the $\mathrm{OE}$ depends on the volume velocity imposed by the free EC wall $\left(\hat{q}_{\text {wall }}^{\text {occl }}\right)$ and the medial EP surface $\left(\hat{q}_{E P}\right)$ and on the acoustic impedance of the occluded EC cavity $\left(\hat{Z}_{E C}^{\text {occl }}\right)$. Since the acoustic impedance $\hat{Z}_{E C}^{o c c l}$ tends to increase when the volume of the occluded EC cavity diminishes [i.e., the cavity becomes stiffer; see Eq. (9)], the decrease in the OE with insertion depth is necessarily explained by the reduction of the volume velocity imposed on the occluded EC cavity. ${ }^{14,15}$ According to Eq. (10), a sufficiently low volume velocity imposed on the occluded EC cavity compared to that imposed in the open case leads to a negative OE [see Fig. 4(b) for the infinite impedance occlusion and Fig. 4(c) for both the infinite impedance occlusion and the foam EP occlusion] despite the increase in the acoustic impedance of the EC cavity in the occluded case compared to the open one. $^{13}$

When the occlusion is created by an infinite impedance $\mathrm{EP}$, the resulting $\mathrm{OE}$ is solely due to the volume velocity imposed by the free EC wall. This volume velocity $\hat{q}_{\text {wall }}^{\text {occl }}$ depends on the surface averaged normal velocity field (i.e., causing compression and rarefaction in the EC cavity) and its vibrating area [see Eq. (6)]. In this case (i.e., infinite impedance EP), the OE is observed to decrease by $28 \mathrm{~dB}$ from shallow to medium insertion and by $19-40 \mathrm{~dB}$ (depending on the frequency) from medium to deep insertion (see Fig. 4).

Figure 5 displays the volume velocity imposed by the free EC wall $\hat{q}_{\text {wall }}^{\text {occl }}$ (for the case of infinite impedance EP but similar in the cases of foam and silicone EPs) and the medial EP surface $\hat{q}_{E P}$ at (a) shallow, (b) medium, and (c) deep insertion and normalized by the volume velocity imposed by the whole EC wall in the open case. The normalization of volume velocities imposed on the occluded EC by that imposed on the open EC is justified by Eq. (10) of the OE computed by the EA model in which $\hat{q}_{\text {wall }}^{\text {occl }}$ and $\hat{q}_{E P}$ are divided by $\hat{q}_{\text {wall }}^{\text {open }}$. All normalized volume velocities displayed in Fig. 5 are lower than $0 \mathrm{~dB}$, meaning that volume velocities imposed by the free EC wall and the medial EP surface are always lower than the volume velocity imposed by the whole EC wall in the open case.

Figure 5 shows that the volume velocity $\hat{q}_{\text {wall }}^{\text {occl }}$ imposed by the free EC wall drastically decreases with insertion depth, as expected. ${ }^{5,17}$ According to Eq. (6), $\hat{q}_{\text {wall }}^{\text {ocl }}$ is computed as the product of the surface average free EC wall normal velocity times the free EC wall area. If the EC wall normal velocity field amplitude were equally distributed over the EC wall surface, the reduction of the volume velocity $\hat{q}_{w a l l}^{o c c l}$ imposed by the free EC wall from the medium insertion case to the deep insertion case would be of $3 \mathrm{~dB}$

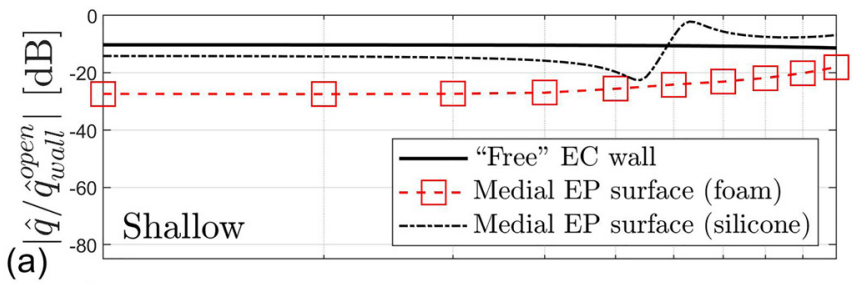

(a)

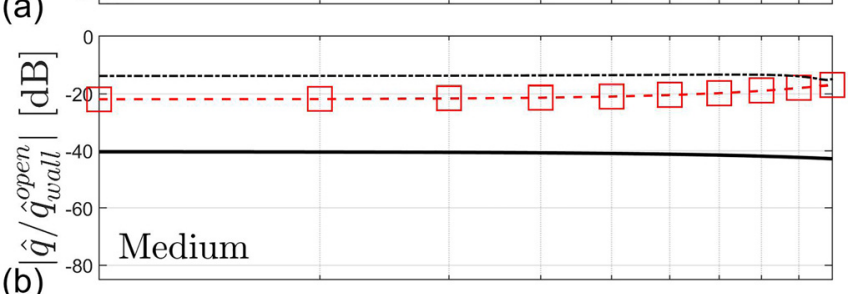

(b)

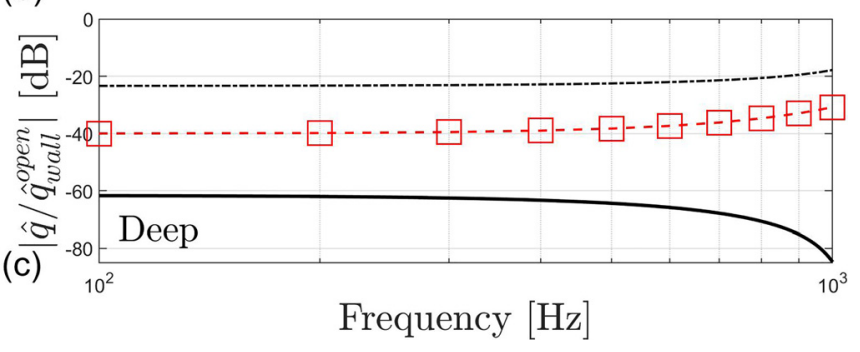

FIG. 5. (Color online) Amplitude in dB (20 $\log _{10}$, Ref. 1) of the volume velocity imposed on the occluded EC cavity by the free EC wall and the medial EP surface (foam and silicone) divided by the volume velocity imposed on the open EC cavity by the whole EC wall at (a) shallow $(6 \mathrm{~mm})$, (b) medium $(12 \mathrm{~mm})$, and (c) deep $(18 \mathrm{~mm})$ insertion computed using the FE model as a function of frequency.

(since the free EC wall surface would be reduced by a factor 2), which is negligible compared to the $20 \mathrm{~dB}$ decrease observed in Fig. 5 at $100 \mathrm{~Hz}$. The reduction of $\hat{q}_{\text {wall }}^{\text {occl with }}$ insertion depth seen in Fig. 5 is therefore mainly due to the decrease in the surface average free EC wall normal velocity with insertion depth whereas the reduction of its vibrating area has little effect. To illustrate the reduction of the EC wall normal velocity with insertion depth, Fig. 6(a) displays the radial component ( $r$-direction, normal to the $\mathrm{EC}$ wall surface) of the structural velocity field in the EC surrounding tissues (i.e., skin, cartilage, and bone) computed using the FE model at $100 \mathrm{~Hz}$. The amplitude of the EC wall normal velocity is maximal at the EC entrance, where the soft tissues are located, and suddenly decreases in the bony part, where the tissues are much less able to deform at low frequency as mentioned by Berger and Kerivan. ${ }^{8}$ The minimum normal velocity does not exactly occur at the tympanic membrane but rather a few millimeters ahead (see the blue region). The centroid position $l_{c, n}$ [see the red " $\mathrm{X}$ " in Fig. $6(a)]$ of the EC wall normal velocity distribution is located at $4.2 \mathrm{~mm}$ from the EC entrance, in the cartilaginous part. This centroid position does not significantly vary with frequency due to the absence of structural modes at low frequency in the present FE model. The current vibration pattern cannot be directly compared to measurement due to the absence of experimental data. This is mainly explained by the difficulty in assessing the distribution of the EC wall vibration (discussed in Sec. III D). However, this pattern is in qualitative agreement with experimental findings, which 


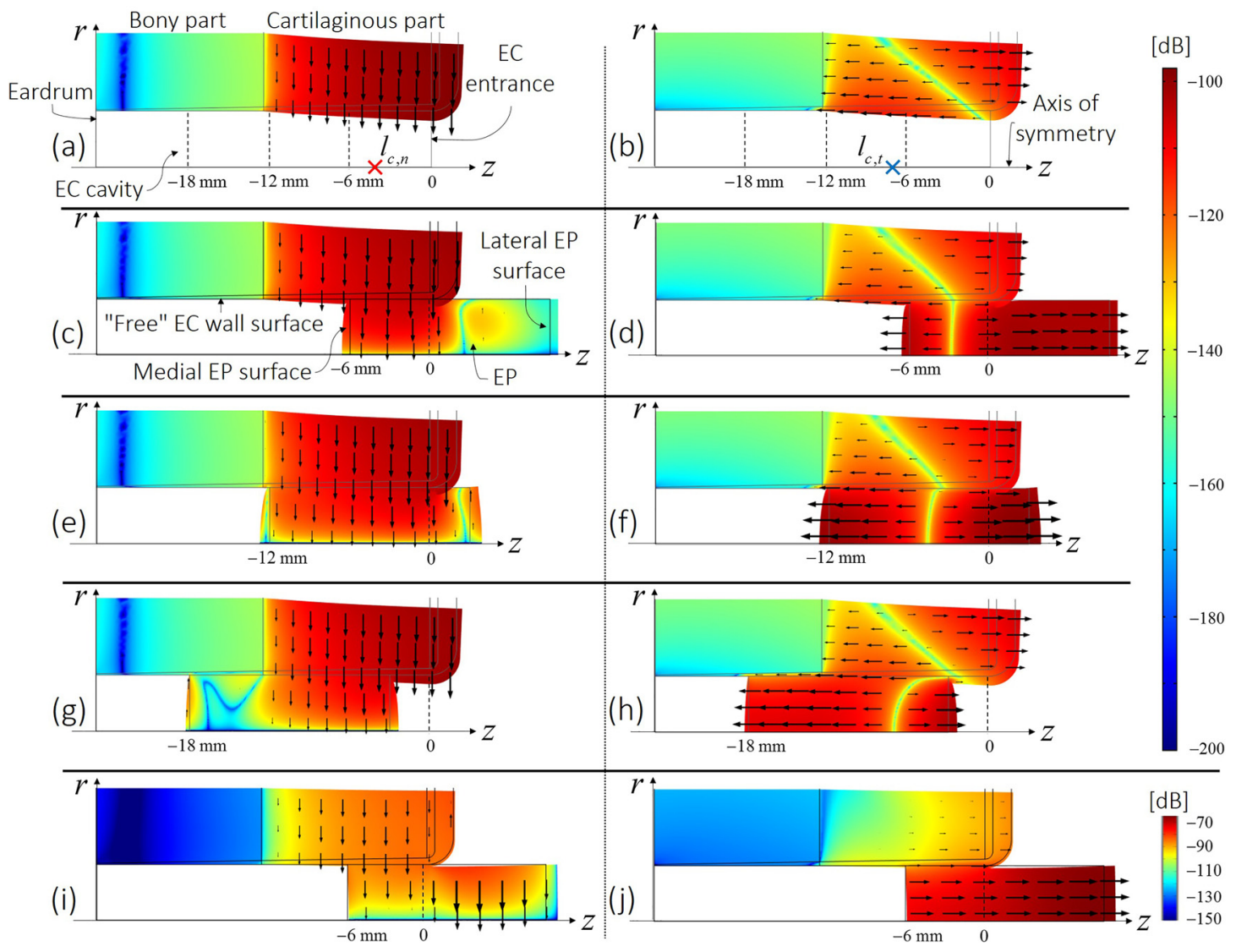

FIG. 6. (Color online) Amplitude in dB $\left[20 \log _{10}\right.$, Ref. $1 \mathrm{~m} \cdot \mathrm{s}^{-1}$ for the colorbar] of the radial ( $r$-direction, left side) and longitudinal ( $z$-direction, right side) structural velocity components of the system computed at $100 \mathrm{~Hz}(t=T / 8$, where $T=1 / f$ is the period) using the FE model. Black arrows represent structural velocity vectors (the longer the arrow, the larger the velocity amplitude). The EC is open [(a) and (b)] or occluded by the silicone EP in shallow [(c) and (d)], medium [(e) and (f)], and deep [(g) and (h)] insertion. Similar results are presented for the shallowly inserted EP at $600 \mathrm{~Hz}$ [(i) and (j)]. In (a) and (b), the red and blue " $\mathrm{X}$ " indicates the centroid position of the EC wall vibration in normal and tangential directions, respectively. In all subplots, the label on the $z$ axis indicates the EC entrance $(z=0 \mathrm{~mm})$ and the three different insertion depths of the medial EP surface $(z \in\{-6,-12,-18 \mathrm{~mm}\})$. A scale factor has been applied in all subplots to highlight the total deformation of the system in the $(r, z)$ plane, and this is evidenced by the appearance of the color maps exceeding the actual dimensions of the extent of the EC and EP in the drawings. Each drawing of the model has been cut in the $r$-direction along the $z$ axis to limit the size of the figure.

suggest that the cartilaginous part of the EC vibrates the most. $^{3,12}$

When the EP is considered, Fig. 4 shows that the associated $\mathrm{OE}$ is higher than that induced by the infinite impedance, depending on the EP type and its insertion depth. At shallow insertion [see Fig. 4(a)], the OE induced by the foam $\mathrm{EP}$ is almost equal to that of the infinite impedance, while the $\mathrm{OE}$ of the silicone $\mathrm{EP}$ is $4-7 \mathrm{~dB}$ higher. At medium insertion [see Fig. 4(b)], the foam EP induces an OE $20 \mathrm{~dB}$ higher ( $28 \mathrm{~dB}$ for the silicone EP) than the infinite impedance. At deep insertion [see Fig. 4(c)], the gap widens since the OE of the foam EP is $23-53 \mathrm{~dB}$ higher (39-66 dB for the silicone EP) than that of the infinite impedance. Contrary to what Hansen ${ }^{14}$ proposed, the presence of the EP does not significantly influence the volume velocity imposed by the free EC wall in the current model ${ }^{18}$ (not shown here). The use of a truncated FE model of small size and the application of current boundary and loading conditions (see Sec. IIC) might lead to a structure stiffer than a real ear. Therefore, the volume velocity imposed by the free EC wall could be less influenced by the coupling with the EP in the present model than in reality. Also, this influence could be greater if the EC surrounding tissues were prestressed. In this study, prestress of EC surrounding tissues by EPs has been neglected (see Sec. II B). Indeed, accounting for prestress could be challenging due to the modeling complexity associated with nonlinear deformation and the lack of knowledge related to constitutive relations governing the mechanical behavior of prestressed EC surrounding tissues. However, the volume velocity imposed by the free EC wall is negligible in the current model compared to that imposed by the EP medial surface at medium and deep insertion [see Figs. 5(b) and 5(c)]. Therefore, the hypothetical influence of the EP on the free EC wall vibration is not of primary importance since it could not explain the influence of the EP on the OE at medium and deep insertion [see Figs. 4(b) and $4(\mathrm{c})]$.

As suggested by Brummund et al., ${ }^{7}$ the influence of the $\mathrm{EP}$ on the $\mathrm{OE}$ is rather directly due to the normal vibration of its medial surface in the $z$-direction. The latter imposes a volume velocity $\hat{q}_{E P}$ on the occluded EC cavity, which is displayed in $\mathrm{dB}$ (normalized by the volume velocity 
imposed by the whole EC wall in the open case) in Fig. 5 at each insertion depth and for both EP types. At a given insertion depth, the higher the volume velocity imposed by the medial EP surface (see Fig. 5), the higher the associated OE (see Fig. 4). According to Fig. 5, the volume velocity imposed by EPs does not significantly vary between shallow and medium insertion, but it decreases by $10-15 \mathrm{~dB}$ at deep insertion. To illustrate this aspect, Fig. 6 displays the structural velocity field ( $r$ - and $z$-directions) in the outer ear occluded by the silicone EP at shallow [(c) and (d)], medium [(e) and (f)], and deep [(g) and (h)] insertion computed at $100 \mathrm{~Hz}$ using the FE model. A scale factor is applied to the deformed structure to highlight the total deformation of the system. Hence, the color maps are seen to exceed the nominal geometrical dimensions of the extent of the EC and EP in the drawings. It is noteworthy that similar structural vibration patterns are obtained with the foam EP and are thus not presented.

According to Figs. 6(d), 6(f), and 6(h), the amplitude of the normal velocity (z-direction) of the medial EP surface decreases by approximately $10 \mathrm{~dB}$ when the silicone EP is inserted in the bony part of the EC (deep insertion), since this portion vibrates much less than the cartilaginous part. It should be noted that volume velocities imposed by the free EC wall and medial EP surfaces are in-phase in the whole frequency range of interest for each EP and insertion (not presented for the sake of conciseness), except around $600 \mathrm{~Hz}$ for the shallowly inserted silicone EP. Indeed, in the latter case, the normal velocity of the EC wall is oriented inward to the EC cavity [see Fig. 6(i)], whereas the normal velocity of the medial EP surface is oriented outward from the EC cavity [see Fig. 6(j)]. Around this frequency, the silicone EP medial surface imposes a volume velocity in phase quadrature with the free EC wall due to a structural mode of the EP coupled to the EC wall. The silicone EP is driven by a longitudinal motion in which its medial and lateral surfaces vibrate in-phase [see Fig. 6(j)]. In addition, the medial EP surface is seen to not vibrate in-phase with the free EC wall surface normal vibration [see Fig. 6(i)]. This resonance is responsible for a $2 \mathrm{~dB}$ decrease in the $\mathrm{OE}$ of the silicone $\mathrm{EP}$ at $600 \mathrm{~Hz}$ due to the phase difference between the vibration of the medial EP surface and that of the free EC wall. A similar phenomenon was expected by Berger and Kerivan ${ }^{8}$ to explain a local minimum in the subjective OE measured on human subjects around $500 \mathrm{~Hz}$ using a deep inserted foam EP. In the current model, such a phenomenon cannot appear in deep insertion because volume velocities imposed by the medial EP surface and the free EC wall are not of the same order of magnitude [see Fig. 5(c)]. As observed in Fig. 5, the volume velocity imposed by the medial EP surface becomes of major importance compared to the volume velocity imposed by the free EC wall when the insertion depth of the EP increases. At shallow insertion, the contribution in volume velocity imposed by both foam and silicone EPs medial surface is negligible, except above $600 \mathrm{~Hz}$ for the silicone EP [see Fig. 4(a)]. This approximation is, however, not valid anymore when the EP insertion depth increases to medium and deep [see Figs. 4(b) and 4(c)] because the contribution of the EP in terms of volume velocity (depending on the EP type) dominates that of the free EC wall and thus cannot be neglected [see Figs. 5(b) and 5(c)].

At medium and deep insertion, these results give support to the suggestion proposed by Bárány ${ }^{3}$ that the vibration of the medial EP surface is of primary importance for the OE. Hence, these results differ from the common assumption that the main source of volume velocity is the free EC wall (even in the occluded case), which originates from the early work of von Bekesy. ${ }^{21,22}$ However, these results explain the experimental observation made by Lee and $\mathrm{Casali}^{23}$ that the influence of the EP type on the OE is little at shallow insertion and increases with insertion depth (see Fig. 4). Indeed, for the EP's influence on the OE to vary according to its material properties, the EP has to be the main source of volume velocity in the occluded EC. In past EA models, ${ }^{5,14,15,17}$ the contribution of the EP in terms of volume velocity was thus generally neglected or hidden in the volume velocity source representing the free EC wall vibration, mainly due to the difficulty in accounting for the EP's vibro-acoustic behavior. ${ }^{14}$

\section{Influence of the EP material properties}

In Sec. III B 1, the silicone EP has been shown to impose a volume velocity $\hat{q}_{E P}$ higher than the foam EP for each insertion depth (see Fig. 5), resulting in a higher OE induced by the silicone EP compared to the foam EP (see Fig. 4). To investigate the influence of the EP material properties (e.g., complex Young's modulus including the structural loss factor, density, and Poisson's ratio) on the volume velocity imposed on the occluded EC cavity by the medial EP surface, a $2^{3}$ full factorial design of experiment has been performed using the FE model. This design of experiment makes it possible to assess the main effect of each parameter as well as the interactions between parameters. The lower level of each material property comes from the foam EP, while the upper level comes from the silicone EP (see Table I in Appendix A).

Results of the design of experiment are shown in detail in Appendix D, while the main conclusions are presented here. For all insertion depths, the design of experiment reveals that, compared to the Poisson's ratio, the Young's modulus and the density of the EP are of minor influence on the volume velocity imposed by the medial EP surface (for the range of variation considered here). The difference in terms of volume velocity imposed by the medial EP surface between the foam and the silicone EP seen in Fig. 5 is thus explained by the Poisson effect. The sensitivity analysis of the $\mathrm{OE}$ to the Poisson's ratio displayed in Fig. 12 (Appendix A) shows the gradual increase in OE with Poisson's ratio, in particular above a value of 0.2 . The Poisson effect corresponds to the medial and lateral expansion of the EP induced by the radial compression imposed by the normal vibration of the EC wall. However, there is no simple relation between the normal vibration applied by 
the EC wall to the circumferential surface of the EP and the vibration of the medial EP surface mainly because the vibration field of the EC wall is not spatially uniform but significantly varies along the EP surface, depending on its insertion depth [see Figs. 6(c), 6(e), and 6(g)]. Figure 7 displays the OE imposed by the "foam" and "silicone" EPs by presuming a Poisson's ratio equal to zero for (a) shallow, (b) medium, and (c) deep insertion. For comparison, the OE induced by the infinite impedance, the foam, and the silicone EPs (with the Poisson effect) are also displayed. The simulated OE obtained presuming zero Poisson's ratio has been computed in narrowband but displayed at selected frequencies only to increase the readability of Fig. 7. For each insertion depth, there is no significant difference in $\mathrm{OE}$ between the foam and the silicone EP when neglecting the Poisson effect. Also, for each insertion depth, the OE of the foam EP is influenced much less by the Poisson effect than that of the silicone EP. This is due to the much lower Poisson's ratio of the foam material $(\nu=0.1)$ compared to the silicone material $(\nu=0.48)$, almost incompressible.

For the medium and deep insertions [see Figs. 7(b) and 7(c)], the OE induced by both EPs without considering the Poisson effect is approximately $20 \mathrm{~dB}$ higher than the $\mathrm{OE}$ induced by the infinite impedance. This difference is

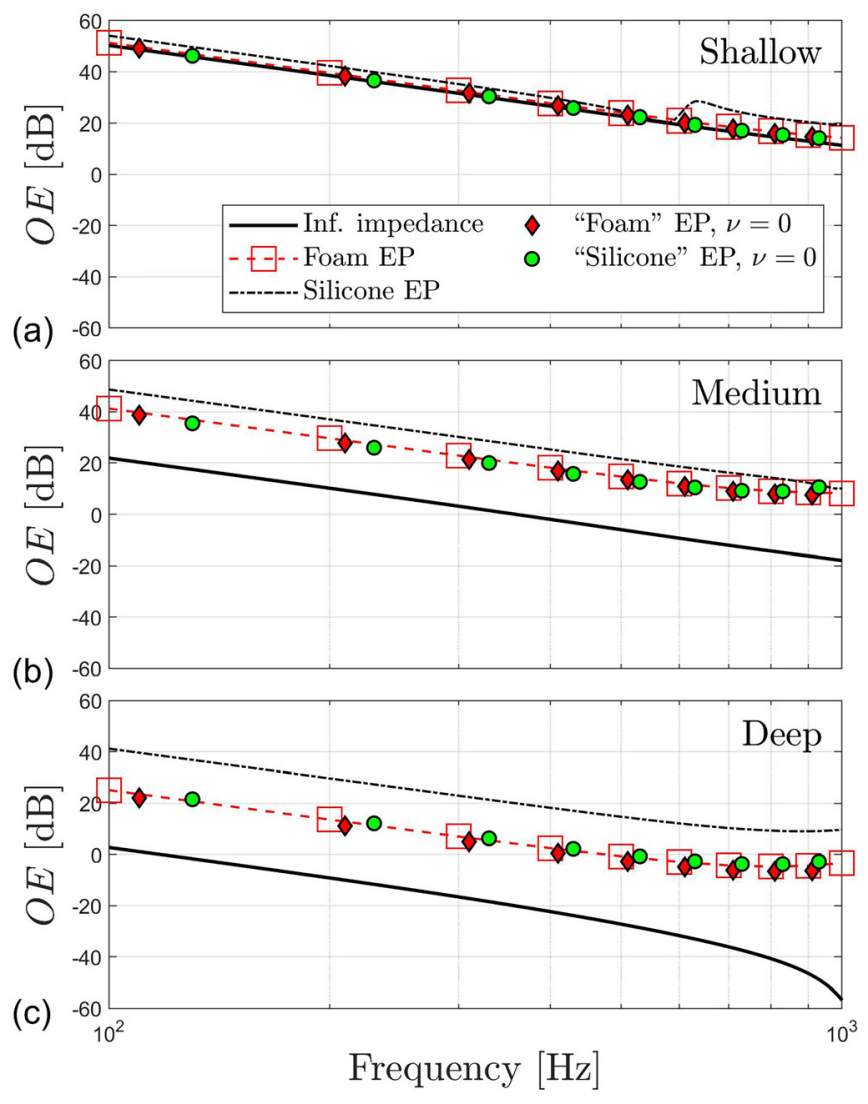

FIG. 7. (Color online) Predicted objective OE induced by a "foam" and a "silicone" EP presuming zero Poisson's ratio $(\nu=0)$ at (a) shallow $(6 \mathrm{~mm})$, (b) medium $(12 \mathrm{~mm})$, and (c) deep $(18 \mathrm{~mm})$ insertion computed using the FE model (in narrowband but displayed at selected frequencies only). For comparison, OE induced by an infinite impedance, a foam EP, and a silicone EP was computed using the FE model and presented in Fig. 4. explained by a second mechanism of contribution of the EP, which corresponds to a longitudinal motion of the EP induced by the tangential component of the EC wall vibration along the $z$-direction. Figure 6(b) displays the longitudinal component ( $z$-direction) of the structural velocity field of the EC surrounding tissues in the open EC. The amplitude of the EC wall tangential velocity (parallel to the EC longitudinal $z$-direction) is at a minimum at the EC entrance, where the longitudinal velocity changes sign [see the green diagonal region in Fig. 6(b)], and then increases up to the mid-length of the cartilaginous part and finally drastically decreases in the bony part. According to Fig. 6(b), the centroid position $l_{c, t}$ (see the blue "X") of the EC wall tangential velocity distribution is located at $7.3 \mathrm{~mm}$ from the EC entrance, farther than $l_{c, n}$. This second mechanism (i.e., longitudinal body motion of the EP) does not depend on the material properties of the EPs in the range of variation considered here and at low frequencies. This mechanism is however decreased with a rigid or very heavy EP (not shown here). In the current model, both mechanisms (i.e., Poisson effect and the longitudinal body motion) of contribution of the EP in terms of volume velocity imposed on the EC cavity act in-phase because the tangential component of the EC wall vibration is oriented toward the eardrum [see Fig. 6(b)] when the normal component is oriented radially inward within the EC cavity [see Fig. 6(a)]. Also, both mechanisms could be expected to influence each other. For example, by causing the EP to expand and contract longitudinally, the Poisson effect of the EP influences the spatial distribution of the tangential velocity of the EC wall, depending on the EP insertion depth [see Figs. 6(d), 6(f), and 6(h)]. In consequence, a change of the EC wall tangential velocity could result in a change in the EP longitudinal body motion. The influence of the Poisson effect on the longitudinal body motion is, however, intricate to distinguish in the volume velocity imposed by the EP on the EC cavity.

\section{Influence of the EC wall vibration distribution on the $\mathrm{OE}$}

In Sec. III B 2, two mechanisms of contribution of the EP to the OE have been highlighted: (i) Poisson effect induced by the normal component of the EC wall vibration and (ii) the longitudinal motion governed by the tangential component of the EC wall vibration. Both mechanisms depend on the vibration of the surrounding EC wall. The influence of the distribution of this vibration is now studied. In Ref. 13, the distribution of the EC wall vibration was varied by applying different sets of loading and boundary conditions while the geometry was fixed. In this study, however, the influence of a geometric parameter on this distribution and consequently on the OE is examined. A geometric parameter that has not been studied in previous work is the transition between the cartilaginous and bony tissues surrounding the EC cavity. This transition is expected to approximately occur at the mid-length of the EC, ${ }^{5}$ but its geometry varies significantly around the EC cavity, as exposed in Sec. II A. Here, three anatomically different 
geometries of the transition between cartilaginous and bony tissues have been constructed using the 2D FE model from an anatomical model of a 3D human outer ear (see Fig. 2). These 2D geometries have been presented in Sec. II A [see Figs. 1(b), 1(c), and 1(d)] and only differ by their transitions between the cartilaginous and bony tissues.

Figure 8 illustrates the structural vibration of the EC wall surrounding tissues computed using the FE model at $100 \mathrm{~Hz}$ for the three transitions: (a) straight, (b) slanted, and (c) curved. It is noteworthy that each drawing of the model has been cut in the $r$-direction along the $z$ axis to limit the size of the figure. Due to this cut, the full geometry of each transition between cartilaginous and bony part is not visible in Fig. 8 but can be seen in Figs. 1(b), 1(c), and 1(d). The first case [see Fig. 8(a)] corresponds to the standard configuration of the FE model used in Secs. III A and IIIB. For each transition, the amplitude of the EC wall vibration decreases from the EC entrance to the eardrum and is significantly lower in the bony part compared to the cartilaginous part. Distributions of the normal and tangential vibrations of the EC wall are characterized by their centroid positions $l_{c, n}$ and $l_{c, t}$ defined from the EC entrance in this work. As shown in Fig. 8, when the transition between cartilaginous and bony tissues moves toward the eardrum, both centroid positions move too: $l_{c, n}$ decreases from -4.5 to $-6.2 \mathrm{~mm}$ and $l_{c, t}$

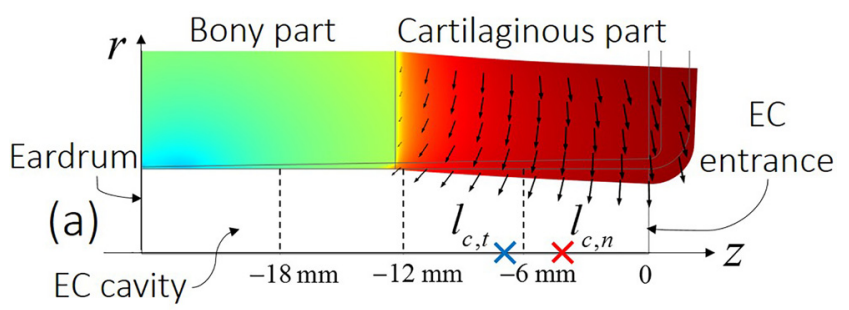

(b)
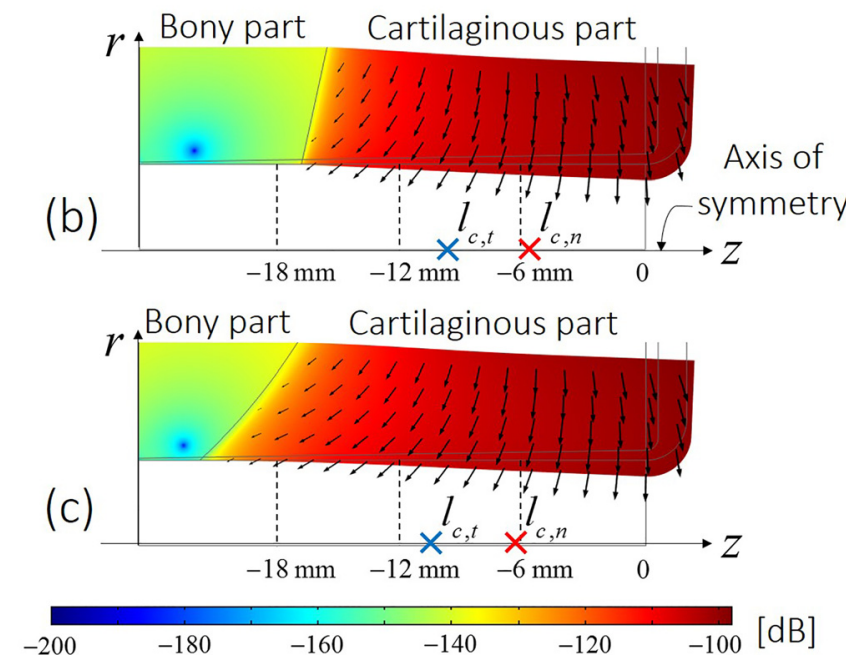

FIG. 8. (Color online) Amplitude in $\mathrm{dB}\left(20 \log _{10}\right.$, Ref. $\left.1 \mathrm{~m} \cdot \mathrm{s}^{-1}\right)$ of the structural velocity of the EC surrounding tissues computed using the FE model at $100 \mathrm{~Hz}(t=T / 8)$ for the three different transitions between cartilaginous and bony tissues of the outer ear: (a) straight, (b) slanted, and (c) curved. Black arrows represent velocity vectors. A scale factor has been applied in all subplots to highlight the total deformation of the system in the $(r, z)$ plane. Each drawing of the model has been cut in the $r$-direction along the $z$ axis to limit the size of the figure. decreases from -7.3 to $-10.6 \mathrm{~mm}$. For the same reasons explained in Sec. III B 1, vibration patterns exhibited here by the two additional anatomical models of the outer ear [see Figs. 8(b) and 8(c)] cannot be compared to experimental data.

Figure 9 displays the OE induced by the infinite impedance, the foam EP, and the silicone EP computed at $100 \mathrm{~Hz}$ as a function of insertion depth (from 6 to $18 \mathrm{~mm}$ ) using the FE model for the three anatomical geometries characterized by their different transitions between cartilaginous and bony tissues of the outer ear: (a) straight, (b) slanted, and (c) curved. At shallow insertion $(6 \mathrm{~mm})$, there is no significant difference in OE between the three different anatomical models. In each case, the OE induced by the infinite impedance is equal to the OE of the foam EP (i.e., negligible longitudinal motion of the EP) and almost equal to the OE of the silicone EP (i.e., little influence of the EP Poisson effect). This means that the volume velocity imposed by the free EC wall governs the OE for each transition, while the contribution of the EP has little influence.

However, substantial variations in OE occur between the three anatomical models when the insertion depth increases. First, the OE induced by the infinite impedance increases between the three transitions, in particular from medium to deep insertion. At deep insertion $(18 \mathrm{~mm})$, the $\mathrm{OE}$ induced by the infinite impedance increases from $3 \mathrm{~dB}$ in the straight transition [see Fig. 9(a)] to $25 \mathrm{~dB}$ in the curved transition [see Fig. 9(c)]. This is explained by the fact that the medial surface of the EP does not enter the portion of the EC wall that is directly backed by bony tissue in

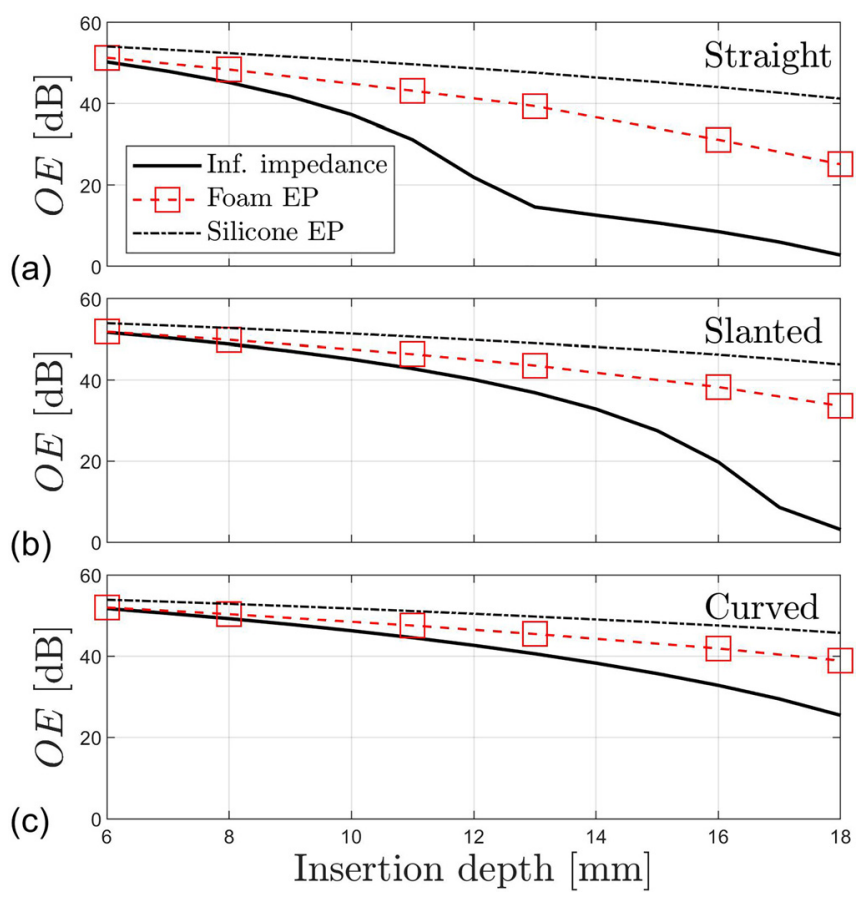

FIG. 9. (Color online) Predicted objective OEs induced by an infinite impedance, a foam EP, and a silicone EP computed at $100 \mathrm{~Hz}$ as a function of the insertion depth using the FE model for the three different transitions between cartilaginous and bony tissues of the outer ear: (a) straight, (b) slanted, and (c) curved. 
the curved transition. The augmentation of the OE induced by the infinite impedance from the straight transition to the curved transition (in particular, at medium and deep insertions) reveals an increase in the contribution of the free EC wall in terms of volume velocity to the OE. This increase is explained by the variation of the EC wall normal vibration distribution that moves toward the eardrum (i.e., the centroid position $l_{c, n}$ diminishes from $-4.5 \mathrm{~mm}$ in the straight transition to $-6.2 \mathrm{~mm}$ in the curved transition). Second, due to the increase in the free EC wall contribution between the three cases, the reduction of the OE with insertion depth decreases. Indeed, the reduction with insertion depth (from 6 to $18 \mathrm{~mm}$ ) of the $\mathrm{OE}$ induced by the foam EP decreases from $26 \mathrm{~dB}$ [see Fig. 9(a)] to $13 \mathrm{~dB}$ [see Fig. 9(c)] and from $13 \mathrm{~dB}$ [see Fig. 9(a)] to $8 \mathrm{~dB}$ [see Fig. 9(c)] for the silicone EP. Third, in consequence to the increase in the free EC wall contribution from the straight transition to the curved transition, the influence of the EP contribution decreases between the three transitions. In consequence, the difference in $\mathrm{OE}$ between the foam and the silicone EP decreases between the three transitions [from 22 to $14 \mathrm{~dB}$ at $18 \mathrm{~mm}$; see Figs. 9(a) and 9(c)]. This means that the influence of the Poisson effect of the EP on the OE diminishes. In addition, the difference in OE between the foam EP (negligible Poisson effect) and the infinite impedance also decreases [from 22 to $14 \mathrm{~dB}$ at $18 \mathrm{~mm}$; see Figs. 9(b) and 9(c)]. This means that the influence of the longitudinal motion of the $\mathrm{EP}$ on the OE also diminishes.

It should be noted that the variation of the EC wall normal vibration distribution influences not only the vibroacoustic behavior of the occluded EC but also that of the open EC, since its acoustic mass is defined between the EC entrance and the centroid position $l_{c, n}$ [see Eq. (5) of the EA model and Appendix B]. However, the variation of $l_{c, n}$ from -4.5 to $-6.2 \mathrm{~mm}$ between the straight and the curved configurations [see Figs. 8(a) and 8(c)] has little influence here $(<2 \mathrm{~dB})$ on the open EC cavity acoustic impedance.

\section{Main limitations and perspectives of the study}

A 2D axi-symmetric FE model initially developed by Brummund et al. ${ }^{7}$ and slightly modified here has been used to predict and investigate the objective OE induced by an EP depending on its material properties and its insertion depth. Since this study focuses on the objective OE, the developed FE model only accounts for the outer ear, while other pathways such as the middle and inner ears have been neglected. If the subjective OE were to be investigated, the bone-conducted transmission through the middle and inner ears could not be neglected because the outer ear pathway does not dominate the hearing by bone-conduction when the $\mathrm{EC}$ is open, as mentioned in the Introduction. As a consequence, the subjective $\mathrm{OE}$ is generally $5-10 \mathrm{~dB}$ lower than the objective $\mathrm{OE}$ due to the difference in bone-conduction pathways. ${ }^{4,5,41}$ However, the current study only aimed at investigating the mechanisms of contribution of EPs on the SPL generated in the occluded EC cavity and particularly exploring and explaining the difference in objective OEs induced by a foam and a silicone EP. Therefore, the perception of this difference and its influence on the acoustic comfort of EP wearers was not investigated in this work.

The present model includes the EC cavity (from its entrance to the tympanic membrane) surrounded by a portion of human tissues (e.g., skin, cartilage, bone). For the purpose of the current study, the FE model presents several simplifications, particularly in terms of geometry, boundary conditions, and loading. A whole head model could alleviate these limitations but loses the main advantage of the current model (i.e., few computational resources and parametric geometrical model). In particular, a whole head model could account for the influence of the stimulation position seen in experimental data below $250 \mathrm{~Hz}$ (see Sec. III A). Another limitation of our 2D FE model was the assumption of negligible prestress in the EC surrounding tissues due to the insertion of the EP. This limitation mainly comes from the lack of knowledge regarding the tissues' mechanical properties under prestress (see Sec. II B). Accounting for prestress could influence the respective contribution of each mechanism highlighted here. Also, prestress could lead to a stiffer system, which could result in lower vibration of the medial EP surface and the free EC wall and therefore a lower OE than what was obtained here. However, in the authors' opinion, the effect of prestress is not expected to invalidate the mechanisms of contribution of EPs highlighted in this paper. The effect of prestress in EPs and EC surrounding tissues should be accounted for when more knowledge about their mechanical behavior is obtained experimentally.

It is worth recalling that the main purpose of the additional anatomical geometries was to study the influence of the EC wall vibration distribution on the OE. Indeed, while the three geometrical transitions between cartilaginous and bony tissues have been derived from a realistic anatomical model of a human outer ear, they represent simplified cases, since the geometry of the model is axi-symmetric. The distribution of the EC wall vibration is also expected to vary with different stimulus types and locations. Such variation might explain the variation in $\mathrm{OE}$ with stimulator position ${ }^{6}$ and could also contribute to the OE inter-individual difference ${ }^{5}$ observed in Sec. III A [see Figs. 3(b) and 3(c)]. In the case of chewing or speaking, the mandibular movement ${ }^{42}$ could affect the influence of the EP to the OE. In addition, the contribution of the EP could also be influenced by the shape of the EC, ear hairs, and wax. Due to the complexity associated with their modeling, these phenomena have not been accounted for in this study.

Although the model compares reasonably well with experimental data of the OE despite its simplifications (see Fig. 3), the knowledge of the spatial distribution of the EC wall vibration in the normal and the tangential directions as well as the vibration of the EP is necessary to confirm experimentally the two mechanisms of the EP contribution exposed in this paper (see Sec. III B). The main reason there is a lack of experimental data related to the vibration of the EC wall and the medial EP surface in human subject is due 
to the remarkable difficulty of performing such measurements. Indeed, the small size of the EC as well as its tortuosity make it difficult to assess in a direct way the 3D spatially distributed EC wall vibration using common measurement methods such as a laser Doppler vibrometer ${ }^{12,43}$ or an accelerometer. ${ }^{25}$ Hansen has proposed a method to estimate the volume velocity imposed on the occluded EC cavity based on the measure of the acoustic pressure in the occluded EC cavity and the knowledge of its acoustic impedance. ${ }^{44}$ However, this method does not make it possible to distinguish the contribution of the medial EP surface from that of the free EC wall. The understanding highlighted in the current paper on the mechanisms of contribution of EPs to the $\mathrm{OE}$ will be helpful to ultimately measure them.

Finally, as a perspective of this work, which focused on investigating and explaining the difference in objective $\mathrm{OE}$ between foam and silicone EPs, an existing concept of material used in other application fields that could theoretically be used to design EPs mitigating the OE is suggested. Since the Poisson's ratio governs the difference in OE between foam and silicone EPs, auxetic metamaterial (negative Poisson's ratio) could make it possible to generate destructive interference between the volume velocity imposed by the free EC wall $\left(\hat{q}_{\text {wall }}^{\text {occl }}\right)$ and that imposed in phase opposition by the medial surface $\left(\hat{q}_{E P}\right)$ of the auxetic EP [i.e., $\hat{q}_{\text {wall }}^{\text {ocl }}=-\hat{q}_{E P}$ in Eq. (10)]. For this solution to reduce the $\mathrm{OE}$ to zero, both volume velocities should have an equal magnitude (i.e., $\left|\hat{q}_{\text {wall }}^{\text {occl }}\right|=\left|\hat{q}_{E P}\right|$ ). In practice, the design of auxetic EPs to reduce the OE would be a difficult task, since it would require knowledge of the EC wall velocity field in both normal and tangential directions, which is likely to differ between individuals and to vary from one boneconducted excitation to another.

\section{CONCLUSION}

This paper has investigated the mechanisms of contribution of EPs to the OE. For this purpose, a 2D axi-symmetric FE model of an outer ear has been used. Simulation results of the OE have been compared to existing data taken from the literature. Finite element simulations have been investigated and interpreted using the "framework" of an EA model associated with the FE model. Using a design of experiment performed on the EP material properties in the FE model, the difference in OE between a silicone and a foam EP has been attributed to a higher Poisson effect for the latter compared to the former, which increases the OE. This Poisson effect is induced by the normal component of the EC wall vibration. The increase in the EP influence on the $\mathrm{OE}$ with insertion depth has been explained by the augmentation of the relative contribution of the medial EP surface in terms of volume velocity compared to that of the free EC wall coupled to the occluded EC cavity. In conjunction with the Poisson effect, a longitudinal motion of the EP induced by the tangential component of the EC wall vibration has been highlighted. Results have shown that this mechanism is independent from the EP material properties in the range of variation considered here (foam or silicone). Both mechanisms depend on the EC wall vibration and its spatial distribution in terms of direction and amplitude. This study has underlined the lack of experimental data related to the vibration of the EC wall and the EP, mainly due to the complexity of such measurements. In the authors' opinion, further work should focus on this experimental aspect. In addition, the current study could be continued using a whole head model that could include a realistic vibratory behavior of the human head. Also, a whole head model could include the middle and inner ears, which would allow for the study of the subjective $\mathrm{OE}$ in addition to the objective OE.

\section{ACKNOWLEDGMENTS}

The authors acknowledge the support of the Natural Sciences and Engineering Research Council of Canada (NSERC) (funding reference number RGPIN-2016-06795). Also, all reviewers are gratefully thanked for their critical and wise comments.

\section{APPENDIX A: SOLID DOMAIN PROPERTIES}

Solid domain properties used in the FE model are summarized in Table I.

\section{APPENDIX B: LOCATION OF THE VOLUME VELOCITY SOURCE IN THE OPEN EC}

In Ref. 13, the authors showed that the location of the volume velocity source representing the $\mathrm{EC}$ wall vibration in the EA model could not be placed arbitrarily in the EC when the latter is open because this location influences the inertial effect of the open EC cavity seen by its wall. In addition, the authors showed that locating the source at the centroid position of the EC wall normal velocity led to EA simulations in good agreement with corresponding FE simulations. The relation between the source location and the centroid position, however, was not justified on a mathematical basis. In the current paper, the reason supporting this relation is detailed.

Consider the cylindrical EC of constant radius $r_{E C}$ and length $l_{E C}$. The EC axis corresponds to the $z$ axis. The eardrum is defined at $z=-l_{E C}$, whereas the EC entrance is defined at $z=0$. Note that in Ref. 13, the eardrum was rather defined at $z=0$ and the EC entrance at $z=l_{E C}$. The normal vibration of the surrounding EC wall is accounted for as a distribution of an infinite number of ideal volume velocity sources along the EC axis. Each source $Q_{i}$

TABLE I. Density $\rho$, Young's modulus $E$, Poisson's ratio v, and structural loss factor $\eta$ of solid domains.

\begin{tabular}{lccll}
\hline \hline Tissue & $\rho\left(\mathrm{kg} \mathrm{m}^{-3}\right)$ & $E(\mathrm{MPa})$ & $v(1)$ & $\eta(1)$ \\
\hline Skin & 1100 & 0.5 & 0.4 & 0.1 \\
Cartilage & 1080 & 7.2 & 0.26 & 0.05 \\
Bone & 1714 & 11316 & 0.3 & 0.01 \\
Foam EP & 220 & 0.1 & 0.1 & 0.5 \\
Silicone EP & 1050 & 0.85 & 0.48 & 0.1 \\
\hline \hline
\end{tabular}


represents an infinitesimal portion of length $\delta_{E C}$ of the EC and imposes a volume velocity $\hat{q}_{i}\left(i \in \mathbb{N}^{*}\right)$ such that the sum of all volume velocities is equal to the volume velocity $\hat{q}_{\text {wall }}^{\text {open }}$ imposed by the whole EC wall (i.e., $\hat{q}_{\text {wall }}^{\text {open }}=\sum_{i=1}^{\infty} \hat{q}_{i}$ ). In addition, each source is located at a position $z=-l_{i}$ $\left(-l_{E C} \leq-l_{i} \leq 0\right)$ corresponding to the position of the infinitesimal portion of EC and neglecting its infinitesimal length $\delta_{E C}$. Applying the principle of superposition (linear system), the acoustic pressure at the eardrum $\left(z=-l_{E C}\right)$ is given by

$$
\hat{p}_{T M, E A}^{\text {open }}=\sum_{i=1}^{\infty} \hat{p}_{T M, i}^{\text {open }}
$$

where $\hat{p}_{T M, i}^{\text {open }}$ is the acoustic pressure generated by a source $\mathrm{Q}_{\mathrm{i}}$ and defined by

$$
\hat{p}_{T M, i}^{\text {open }}=\hat{Z}_{E C, i}^{\text {open }} \times \hat{q}_{i},
$$

where $\hat{Z}_{E C, i}^{\text {open }}$ represents the acoustic impedance of the open EC cavity seen by a source $\mathrm{Q}_{\mathrm{i}}$. According to Eq. (3), this acoustic impedance can be simplified to

$$
\hat{Z}_{E C, i}^{\text {open }} \approx j \omega\left(L_{E C, i}^{\text {open }}+L_{\text {rad }}\right)
$$

where $L_{\text {rad }}$ represents the acoustic mass of radiation at the EC entrance and $L_{E C, i}^{\text {open }}$ the acoustic mass of the open EC. Note that in Ref. $13, L_{E C, i}^{\text {open }}$ was rather referred to as $L_{u}$. The latter is defined by $L_{E C, i}^{\text {open }}=-\rho_{0} l_{i} / S_{E C}$ with the air density $\rho_{0}$ and the EC cross section area $S_{E C}=\pi r_{E C}^{2}$. Substituting Eqs. (B2) and (B3) into Eq. (B1), the acoustic pressure at the eardrum can be rewritten as

$$
\begin{aligned}
\hat{p}_{T M, E A}^{\text {open }} & =\sum_{i=1}^{\infty} j \omega\left(L_{\text {rad }}+L_{u}^{i}\right) \times \hat{q}_{i} \\
& =\sum_{i=1}^{\infty} j \omega L_{\text {rad }} \times \hat{q}_{i}+\sum_{i=1}^{\infty} j \omega L_{u}^{i} \times \hat{q}_{i} \\
& =j \omega L_{\text {rad }} \times \hat{q}_{\text {wall }}^{\text {open }}+\frac{j \omega \rho_{0}}{S_{E C}} \sum_{i=1}^{\infty}-l_{i} \times \hat{q}_{i} \\
& =j \omega L_{\text {rad }} \times \hat{q}_{\text {wall }}^{\text {open }}+\frac{j \omega \rho_{0}}{S_{E C}}\left(\sum_{i=1}^{\infty} \frac{-l_{i} \times \hat{q}_{i}}{\hat{q}_{\text {wall }}^{\text {open }}}\right) \times \hat{q}_{\text {wall }}^{\text {open }}
\end{aligned}
$$

From the latter equation, one can recognize an expression of the centroid position $l_{c, n}$ of the EC wall normal vibration given by $l_{c, n}=\sum_{i=1}^{\infty}-l_{i} \times \hat{q}_{i} / \hat{q}_{\text {wall }}^{\text {open }}$.

\section{APPENDIX C: VERIFICATION OF THE ACCURACY OF THE EA FRAMEWORK}

To investigate and interpret FE simulations in the EA framework, the latter must provide accurate approximations of the former. In Fig. 10, the OE computed using Eq. (7) of the EA framework is displayed, superimposed with $\mathrm{OE}$ computed using the FE model. It is noteworthy that EA

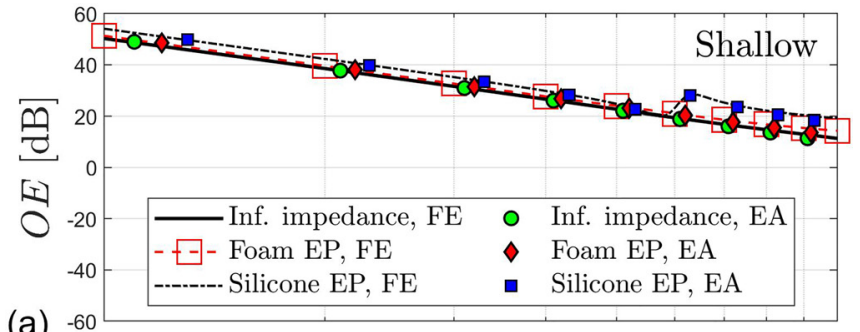

(a)

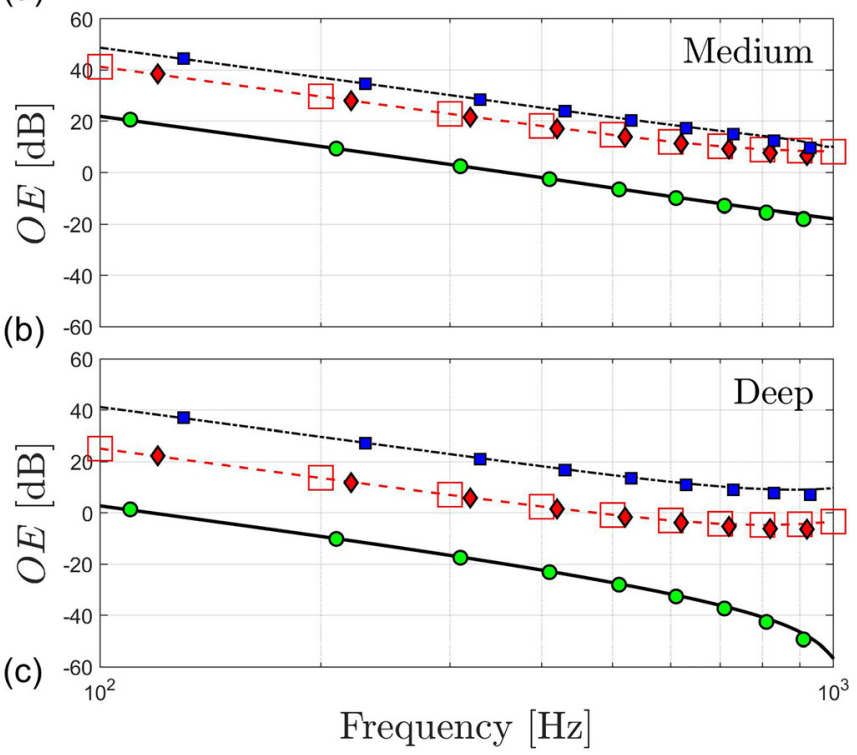

FIG. 10. (Color online) Predicted objective OE induced by an infinite impedance, a foam EP, and a silicone EP at (a) shallow (6 mm), (b) medium $(12 \mathrm{~mm})$, and (c) deep $(18 \mathrm{~mm})$ insertion computed using both the FE model and EA model (in narrowband but displayed at selected frequencies only).

simulations are displayed at selected frequency points rather than in narrowband to increase the readability of Fig. 10. As expected, approximations of the OE obtained using the EA model are in good agreement with FE simulations in all cases (foam, silicone, and infinite impedance EP) and at all insertion depths. This verifies the accuracy of the EA framework, despite its simplifications and assumptions (see Sec. II E).

\section{APPENDIX D: RESULTS OF THE DESIGN OF EXPERIMENT}

Figure 11 displays the influence of the EP material properties on the volume velocity $\hat{q}_{E P}$ imposed by its medial surface for (a) shallow, (b) medium, and (c) deep insertion. Results are presented in terms of variation $\Delta \hat{q}_{E P}$ in $\mathrm{dB}$ from the mean value $(0 \mathrm{~dB})$ of the volume velocity imposed by the medial EP surface computed from the $2^{3}=8$ configurations of the design of experiment. A positive variation means that the parameter (or the couple of parameters) tends to increase the volume velocity imposed by the EP on the occluded EC cavity, whereas negative variation means the opposite. Most important effects only are presented in Fig. 11. For all insertion depths, the Young's modulus and the density of the EP are seen to be of minor influence on the volume velocity imposed by the medial EP surface 


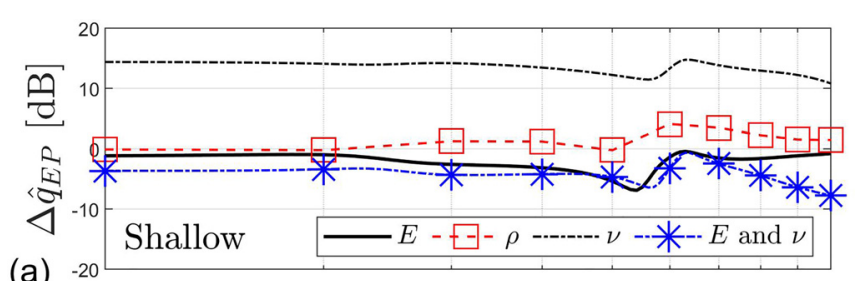

(a)

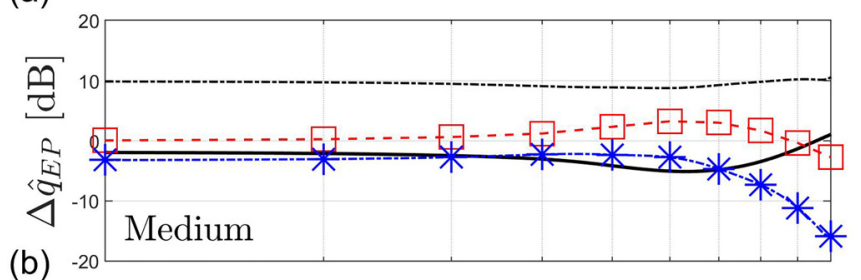

(b)

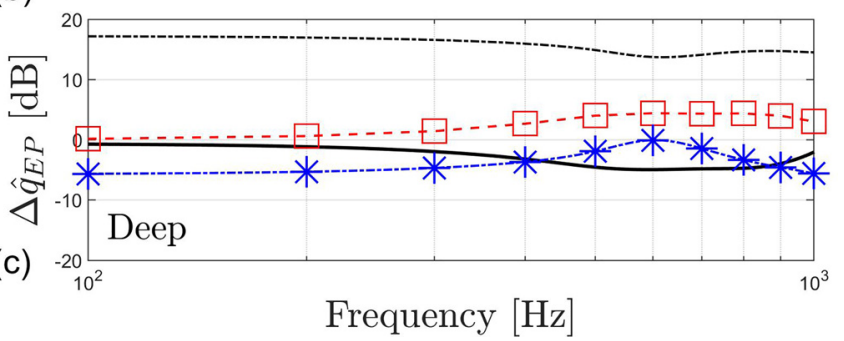

FIG. 11. (Color online) Variation in $\mathrm{dB}\left(20 \log _{10}\right.$, Ref. $\left.1 \mathrm{~m}^{3} \mathrm{~s}^{-1}\right)$ from the mean value of the volume velocity $\hat{q}_{E P}$ imposed by the medial EP surface as computed using the FE model. The results derive from the design of experiment performed on the EP material properties at (a) shallow $(6 \mathrm{~mm})$, (b) medium $(12 \mathrm{~mm})$, and (c) deep $(18 \mathrm{~mm})$ insertion as a function of frequency.

compared to the Poisson's ratio. Indeed, the Poisson effect is the only one that substantially moves the curves from the $0 \mathrm{~dB}$ line, by from 10 to $17 \mathrm{~dB}$ across conditions depending upon frequency. The Poisson effect thus governs the difference in contribution between a foam and a silicone EP. Both the Young's modulus (main effect) and the interaction between the Young's modulus and the Poisson's ratio (interaction effect) tend to decrease the volume velocity imposed by the medial EP surface. This is explained by the bulk modulus of the EP defined in $2 \mathrm{D}$ by $K=E /[2(1-\nu)]$, which measures the resistance of the EP to compression. The bulk modulus of the EP increases with both Young's modulus and Poisson's ratio, which in consequence constrains the normal vibration of the EC wall portion coupled to the EP and therefore reduces the volume velocity imposed by the medial EP surface by the Poisson effect. The effect solely due to Poisson's ratio accounts for the influence of the bulk modulus and the Poisson effect, both of which act in opposition to each other on $\hat{q}_{E P}$. However, since the effect due to Poisson's ratio is positive and significant, it is concluded that the Poisson effect largely dominates the effect of the bulk modulus in the design of experiment.

In complement to Fig. 11, Fig. 12 displays the $\mathrm{OE}$ induced for several Poisson's ratios for a silicone EP. For all insertion depths, the overall amplitude of the OE is seen to increase with Poisson's ratio, confirming the preponderant role of the Poisson effect in the difference in OE between a foam $(\nu=0.1)$ and a silicone $(\nu=0.48) \mathrm{EP}$. The increase in the EP Poisson's ratio from 0 to 0.2 increases the $\mathrm{OE}$ by

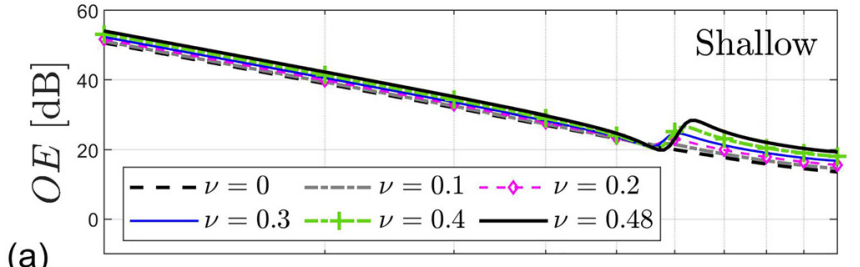

(a)

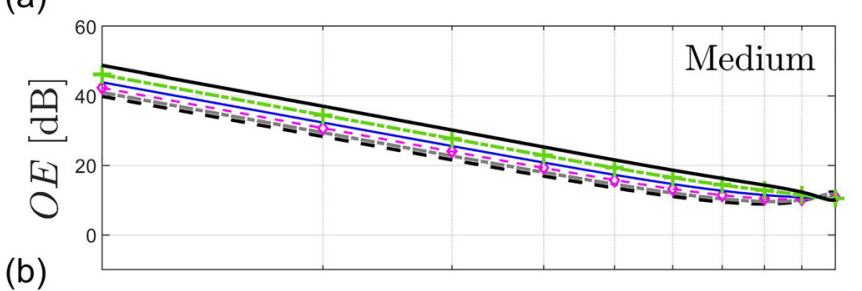

(b)

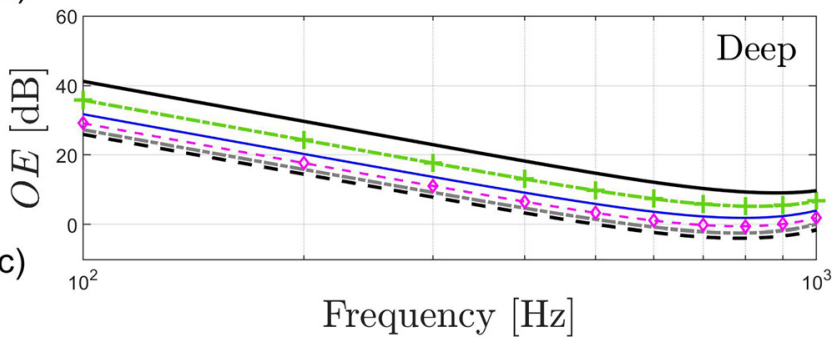

FIG. 12. (Color online) Predicted objective OE induced for several Poisson's ratios for a silicone EP at (a) shallow $(6 \mathrm{~mm})$, (b) medium $(12 \mathrm{~mm})$, and (c) deep $(18 \mathrm{~mm})$ insertion as a function of frequency.

approximately $3 \mathrm{~dB}$ for all insertion depths. However, the increase in the EP Poisson's ratio from 0.2 to 0.48 increases the $\mathrm{OE}$ by approximately $6 \mathrm{~dB}$ (above the resonance frequency around $600 \mathrm{~Hz}), 8 \mathrm{~dB}$, and $12 \mathrm{~dB}$ for shallow, medium, and deep insertion, respectively. The influence of the Poisson's ratio is therefore not linear and varies with insertion depth.

${ }^{1}$ E. H. Berger, "Hearing protection devices," in The Noise Manual, revised 5th ed., edited by E. H. Berger and M. Layne (AHIA, Fairfax, VA, 2003), pp. 379-454.

${ }^{2}$ O. Doutres, F. Sgard, J. Terroir, N. Perrin, C. Jolly, C. Gauvin, and A. Negrini, "A critical review of the literature on comfort of hearing protection devices: Definition of comfort and identification of its main attributes for earplug types," Int. J. Audiol. 58, 824-833 (2019).

${ }^{3}$ E. Bárány, "A contribution to the physiology of bone conduction," Acta Otolaryngol. 26(Suppl. 26), 1-223 (1938).

${ }^{4} \mathrm{E}$. H. Huizing, "Bone conduction-The influence of the middle ear," Acta Oto-Laryngol 155, 1-99 (1960).

${ }^{5}$ S. Stenfelt and S. Reinfeldt, "A model of the occlusion effect with boneconducted stimulation," Int. J. Audiol. 46(10), 595-608 (2007).

${ }^{6} \mathrm{~S}$. Reinfeldt, S. Stenfelt, and B. Håkansson, "Estimation of bone conduction skull transmission by hearing thresholds and ear-canal sound pressure," Hear. Res. 299, 19-28 (2013).

${ }^{7}$ M. Brummund, F. Sgard, Y. Petit, F. Laville, and H. Nélisse, “An axisymmetric finite element model to study the earplug contribution to the bone conduction occlusion effect," Acta Acust. united Acust. 101(4), 775-788 (2015).

${ }^{8}$ E. H. Berger and J. E. Kerivan, "Influence of physiological noise and the occlusion effect on the measurement of real-ear attenuation at threshold," J. Acoust. Soc. Am. 74(1), 81-94 (1983).

${ }^{9}$ D. J. Brown-Rothwell, "The comfort of earplugs-Basis for a descriptive model," master's thesis, University of Southampton, Southampton, UK, 1986.

${ }^{10} \mathrm{~S}$. Conrad and A. Rout, "Perceived occlusion and comfort in receiver-inthe-ear hearing aids," Am. J. Audiol. 22(2), 283-290 (2013). 
${ }^{11}$ J. Terroir, N. Perrin, P. Wild, O. Doutres, F. Sgard, C. Gauvin, and A. Negrini, "Assessing the comfort of earplugs: Development and validation of the French version of the COPROD questionnaire," Ergonomics 64, 912-914 (2021).

${ }^{12}$ S. Stenfelt, T. Wild, N. Hato, and R. L. Goode, "Factors contributing to bone conduction: The outer ear," J. Acoust. Soc. Am. 113(2), 902-913 (2003).

${ }^{13} \mathrm{~K}$. Carillo, O. Doutres, and F. Sgard, "Theoretical investigation of the low frequency fundamental mechanism of the objective occlusion effect induced by bone-conducted stimulation," J. Acoust. Soc. Am. 147(5), 3476-3489 (2020).

${ }^{14}$ M. O. Hansen, "Occlusion effects-Part II-A study of the occlusion effect mechanism and the influence of the earmould properties," Ph.D. thesis, Technical University of Denmark, Kongens Lyngby, Denmark, 1998.

${ }^{15}$ T. Zurbrügg, A. Stirnemannn, M. Kuster, and H. Lissek, "Investigations on the physical factors influencing the ear canal occlusion effect caused by hearing aids," Acta Acust. united Acust. 100(3), 527-536 (2014).

${ }^{16} \mathrm{~K}$. Carillo, O. Doutres, and F. Sgard, "Numerical investigation of the fundamental low frequency mechanisms of the objective occlusion effect," in Proceedings of the 26th International Congress on Sound and Vibration (ICSV26), Montreal, Canada (July 7-11, 2019).

${ }^{17}$ J. Schroeter and C. Poesselt, "The use of acoustical test fixtures for the measurement of hearing protector attenuation. Part II: Modeling the external ear, simulating bone conduction, and comparing test fixture and realear data," J. Acoust. Soc. Am. 80(2), 505-527 (1986).

${ }^{18} \mathrm{~F}$. Sgard, K. Carillo, and, and O. Doutres, "A 2D axisymmetric finite element model to assess the contribution of in-ear hearing protection devices to the objective occlusion effect," in Proceedings of Internoise, Madrid, Spain (June 16-19, 2019), Vol. 259, pp. 2494-2505.

${ }^{19}$ M. Brummund, F. Sgard, Y. Petit, and F. Laville, "Three-dimensional finite element modeling of the human external ear: Simulation study of the bone conduction occlusion effect," J. Acoust. Soc. Am. 135(3), 1433-1444 (2014).

${ }^{20} \mathrm{~K}$. Lee, "Effects of earplug material, insertion depth, and measurement technique on hearing occlusion effect," Ph.D. thesis, Virginia Polytechnic Institute and State University, Blacksburg, VA, 2011.

${ }^{21}$ G. Békésy, "Zur Theorie des Hörens bei der Schallaufnahme durch Knochenleitung" ("On the theory of hearing when absorbing sound through bone conduction"), Ann Phys. 13, 111-136 (1932).

${ }^{22} \mathrm{G}$. Von Békésy, Experiments in Hearing (McGraw-Hill, New York, 1960).

${ }^{23} \mathrm{~K}$. Lee and J. G. Casali, "Investigation of the auditory occlusion effect with implications for hearing protection and hearing aid design," Proc. Hum. Factors Ergon. Soc. Annu. Meet. 55(1), 1783-1787 (2011).

${ }^{24}$ N. A. Watson and R. S. Gales, "Bone-conduction threshold measurements: Effects of occlusion, enclosures, and masking devices," J. Acoust. Soc. Am. 14(4), 207-215 (1943).

${ }^{25}$ Y. Chang, N. Kim, and S. Stenfelt, "The development of a whole-head human finite-element model for simulation of the transmission of boneconducted sound," J. Acoust. Soc. Am. 140(3), 1635-1651 (2016).

${ }^{26}$ H. Xu, F. Sgard, E. Wagnac, and J. De Guise, "Development of an entire human head finite element model based on in-vivo medical images for investigation of sound transmission," in Proceedings of the 26th International Congress on Sound and Vibration (ICSV26), Montreal, Canada (July 7-11, 2019).

${ }^{27}$ J. Tonndorf, "Bone conduction: Studies in experimental animals," Acta Oto-Laryngol. 213, 1-132 (1966)

${ }^{28}$ L. S. Alvord and B. L. Farmer, "Anatomy and orientation of the human external ear,” J. Am. Acad. Audiol. 8, 383-390 (1997).
${ }^{29}$ R. J. Oliveira and G. Hoeker, "Ear canal anatomy and activity," Semin. Hear. 24(4), 265-276 (2003).

${ }^{30} \mathrm{E}$. H. Berger, "'Calibrating' the insertion depth of roll-down foam earplugs," in Proceedings of Meetings on Acoustics ICA2013, Montréal, Canada (June 2-7, 2013).

${ }^{31}$ S. Benacchio, O. Doutres, A. Varoquaux, É. Wagnac, A. Le Troter, V. Callot, and F. Sgard, "Use of magnetic resonance image registration to estimate displacement in the human earcanal due to the insertion of in-ear devices," J. Acoust. Soc. Am. 146(4), 2452-2465 (2019).

${ }^{32} \mathrm{C}$. James, "Finite element modeling and exploration of double hearing protection systems," master's thesis, Virginia Polytechnic Institute and State University, Blacksburg, Virginia, 2006.

${ }^{33}$ ISO 18437-5:2011, "Mechanical vibration and shock-Characterization of the dynamic mechanical properties of visco-elastic materials-Part 5: Poisson ratio based on comparison between measurements and finite element analysis" (International Organization for Standardization, Geneva, Switzerland, 2011).

${ }^{34}$ F. Sgard, H. Nélisse, J. Boutin, F. Laville, J. Voix, and, and M.-A. Gaudreau, "Finite element modeling for the evaluation of sound attenuation of hearing protectors," in Proceedings of Euronoise 2009, Edinburgh, UK (October 26-28, 2009).

${ }^{35}$ F. Sgard, H. Nélisse, M.-A. Gaudreau, J. Boutin, J. Voix, and F. Laville, "Étude de la transmission sonore à travers les protecteurs auditifs et application d'une méthode pour évaluer leur efficacité effective en milieu de travail—Partie 2: Étude préliminaire d'une modélisation des protecteurs auditifs par éléments finis" "'Study of sound transmission through hearing protectors and application of a method to assess their effective efficacy in the workplace - Part 2: Preliminary study of a modeling of hearing protectors by finite elements"), IRSST Report R-680 (Institut de recherche Robert-Sauvé en santé et en sécurité du travail, Montreal, Canada, 2010). ${ }^{36} \mathrm{H}$. Hudde and A. Engel, "Measuring and modeling basic properties of the human middle ear and ear canal. Part III: Eardrum impedances, transfer functions and model calculations," Acust. Acta Acust. 84, 1091-1109 (1998).

${ }^{37}$ E. A. G. Shaw and M. R. Stinson, "The human external and middle ear: Models and concepts," in Mechanics of Hearing: Proceedings of the IUTAM/ICA Symposium Held at Delft University of Technology, The Netherlands, 13-15 July 1983, edited by E. de Boer and M. A. Viergever (University Press, Delft, Netherlands, 1983), pp. 3-10.

${ }^{38}$ S. Stenfelt, N. Hato, and R. L. Goode, "Factors contributing to bone conduction: The middle ear," J. Acoust. Soc. Am. 111(2), 947-959 (2002).

${ }^{39} \mathrm{~N}$. Atalla and F. Sgard, Finite Element and Boundary Methods in Structural Acoustics and Vibration (CRC, Boca Raton, FL, 2015).

${ }^{40} \mathrm{M}$. Brummund, "Study of the occlusion effect induced by an earplug: Numerical modelling and experimental validation," Ph.D. thesis, École de Technologie Supérieure, Montreal, Canada, 2014.

${ }^{41}$ S. Reinfeldt, S. Stenfelt, T. Good, and B. Håkansson, "Examination of bone-conducted transmission from sound field excitation measured by thresholds, ear-canal sound pressure, and skull vibrations," J. Acoust. Soc. Am. 121(3), 1576-1587 (2007).

${ }^{42}$ S. Darkner, R. Larsen, and R. R. Paulsen, "Analysis of deformation of the human ear and canal caused by mandibular movement," Med. Image Comput. Comput. Assist. Interv. 10, 801-808 (2007).

${ }^{43}$ U. Vogel, T. Zahnert, G. Hofmann, and K.-B. Huettenbrink, "Approach to evidence of middle ear occlusion effect by laser vibrometry," Proc. SPIE Int. Soc. Opt. Eng. 2927, 1-8 (1996).

${ }^{44}$ M. O. Hansen and M. R. Stinson, "Air conducted and body conducted sound produced by own voice," Can. Acoust. 26(2), 11-19 (1998). 